\title{
Clusters of eigenvalues for the magnetic Laplacian with Robin condition
}

\author{
Magnus Goffeng, ${ }^{1, a)}$ Ayman Kachmar, ${ }^{2}$ and Mikael Persson Sundqvist ${ }^{3}$ \\ ${ }^{1}$ Department of Mathematical Sciences, Chalmers University of Technology \\ and the University of Gothenburg, SE-412 96 Gothenburg, Sweden \\ ${ }^{2}$ Department of Mathematics, Faculty of Sciences, Lebanese University, Hadath, Lebanon \\ ${ }^{3}$ Department of Mathematical Sciences, Lund University, Box 118, SE-221 00 Lund, Sweden
}

(Received 6 November 2015; accepted 9 June 2016; published online 28 June 2016)

\begin{abstract}
We study the Schrödinger operator with a constant magnetic field in the exterior of a compact domain in Euclidean space. Functions in the domain of the operator are subject to a boundary condition of the third type (a magnetic Robin condition). In addition to the Landau levels, we obtain that the spectrum of this operator consists of clusters of eigenvalues around the Landau levels and that they do accumulate to the Landau levels from below. We give a precise asymptotic formula for the rate of accumulation of eigenvalues in these clusters, which is independent of the boundary condition. Published by AIP Publishing. [http://dx.doi.org/10.1063/1.4954500]
\end{abstract}

\section{INTRODUCTION}

Magnetic Schrödinger operators in domains with boundaries appear in several areas of physics. For example, the Ginzburg-Landau theory of superconductors, the theory of Bose-Einstein condensates, and of course the study of edge states in quantum mechanics. We refer the reader to Refs. 1, 10, and 16 for details and additional references on the subject. From the point of view of spectral theory, the presence of boundaries has an effect similar to that of perturbing the magnetic Schrödinger operator by an electric potential. In both cases, the essential spectrum consists of the Landau levels and the discrete spectrum form clusters of eigenvalues around the Landau levels. Several papers are devoted to the study of different aspects of these clusters of eigenvalues in domains with or without boundaries. For results in the semi-classical context, see Refs. 11, 12, 14, 17, and 18. In case of domains with boundaries, see Refs. 21 and 22. Analogous results have been obtained in the context of perturbations by electric fields in Ref. 23 . The results in this paper correct a gap in the proof of the main result in Ref. 21 and extend these results to Robin boundary conditions.

Let us consider a compact domain $K \subset \mathbb{R}^{2 d}$ with a Lipschitz boundary. Let us denote by $K^{\circ}$ the interior of $K, \Omega=\mathbb{R}^{2 d} \backslash K$ and $\partial \Omega$ the common boundary of $\Omega$ and $K$. Given a real valued function $\tau \in L^{\infty}(\partial \Omega, \mathbb{R})$ and a positive constant $b$ (the intensity of the magnetic field), we define the Schrödinger operator $L_{\Omega, b}^{\tau}$ with domain $D\left(L_{\Omega, b}^{\tau}\right)$ as follows:

$$
\begin{gathered}
D\left(L_{\Omega, b}^{\tau}\right)=\left\{u \in L^{2}(\Omega):\left(\nabla-i b \mathbf{A}_{0}\right)^{j} u \in L^{2}(\Omega), j=1,2,\right. \\
\left.v_{\Omega} \cdot\left(\nabla-i b \mathbf{A}_{0}\right) u+\tau u=0 \quad \text { on } \partial \Omega\right\}, \\
L_{\Omega, b}^{\tau} u=-\left(\nabla-i b \mathbf{A}_{0}\right)^{2} u \quad \forall u \in D\left(L_{\Omega, b}^{\tau}\right) .
\end{gathered}
$$

Here, $\mathbf{A}_{0}$ is the magnetic potential in the symmetric gauge defined by

$$
\mathbf{A}_{0}\left(x_{1}, x_{2}, \ldots, x_{2 d}\right)=\frac{1}{2}\left(-x_{2}, x_{1}, \ldots,-x_{2 d}, x_{2 d-1}\right),
$$

and $v_{\Omega}$ is the unit outward normal vector of the boundary $\partial \Omega$. We also introduce the boundary Neumann and Robin differential notations

\footnotetext{
a)Electronic addresses: goffeng@ @ chalmers.se; ayman.kashmar@gmail.com; and mickep@maths.lth.se
} 


$$
\partial_{N}=v_{\Omega} \cdot\left(\nabla-i b \mathbf{A}_{0}\right) \quad \text { and } \quad \partial_{R}=\partial_{N}+\tau=v_{\Omega} \cdot\left(\nabla-i b \mathbf{A}_{0}\right)+\tau .
$$

The operator $L_{\Omega, b}^{\tau}$ is actually the Friedrichs self-adjoint extension in $L^{2}(\Omega)$ associated with the semi-bounded quadratic form

$$
\mathfrak{l}_{\Omega, b}^{\tau}(u)=\int_{\Omega}\left|\left(\nabla-i b \mathbf{A}_{0}\right) u\right|^{2} d x+\int_{\partial \Omega} \tau|u|^{2} d S,
$$

defined for all functions $u$ in the form domain

$$
D\left(\mathfrak{l}_{\Omega, b}^{\tau}\right)=H_{\mathbf{A}_{0}}^{1}(\Omega)=\left\{u \in L^{2}(\Omega):\left(\nabla-i b \mathbf{A}_{0}\right) u \in L^{2}(\Omega)\right\} \subseteq H_{\mathrm{loc}}^{1}(\Omega) .
$$

We introduce the (multidimensional) Landau levels $\Lambda_{q}, q \in \mathbb{N}$, as

$$
\Lambda_{q}:=(2(q-1)+d) b, \quad q \in \mathbb{N} \backslash\{0\}, \quad \Lambda_{0}:=-\infty .
$$

The name is motivated by the fact that these numbers (for $q \in \mathbb{N} \backslash\{0\}$ ) are the eigenvalues of the Landau Hamiltonian in $\mathbb{R}^{2 d}$, see Section II B.

We are now able to state the first main result, concerning the essential spectrum of $L_{\Omega, b}^{\tau}$ together with the non-accumulation of eigenvalues to the Landau levels from above.

Theorem 1.1. Let $\Omega \subset \mathbb{R}^{2 d}$ be a compactly complemented Lipschitz domain and $\tau \in L^{\infty}(\partial \Omega, \mathbb{R})$. The essential spectrum of the operator $L_{\Omega, b}^{\tau}$ consists of the Landau levels,

$$
\sigma_{\text {ess }}\left(L_{\Omega, b}^{\tau}\right)=\left\{\Lambda_{q}: q \in \mathbb{N} \backslash\{0\}\right\} .
$$

Moreover, for all $\varepsilon \in(0, b)$ and $q \in \mathbb{N} \backslash\{0\}$, the spectrum of $L_{\Omega, b}^{\tau}$ in the open interval $\left(\Lambda_{q}, \Lambda_{q}+\varepsilon\right)$ is finite.

Next, we restrict our attention to the case that $\partial \Omega$ is $C^{\infty}$ and $\tau \in C^{\infty}(\partial \Omega, \mathbb{R})$. For $\varepsilon>0$, we let $N\left(\Lambda_{q-1}, \Lambda_{q}-\varepsilon, L_{\Omega, b}^{\tau}\right)$ denote the number of eigenvalues of $L_{\Omega, b}^{\tau}$ in the open interval $\left(\Lambda_{q-1}, \Lambda_{q}-\varepsilon\right)$, counting multiplicity. We also denote by $\operatorname{Cap}(K)$ the logarithmic capacity of the domain $K=$ $\mathbb{R}^{2} \backslash \Omega$.

Theorem 1.2. Let $\Omega \subset \mathbb{R}^{2 d}$ be a compactly complemented domain with a smooth boundary and $\tau \in C^{\infty}(\partial \Omega, \mathbb{R})$.

(A) Assume $d=1$. For all $q \in \mathbb{N} \backslash\{0\}$, denoting by $\left\{\ell_{j}^{(q)}\right\}_{j \in \mathbb{N}}$ the nondecreasing sequence of eigenvalues of $L_{\Omega, b}^{\tau}$ (counting multiplicities) in the interval $\left(\Lambda_{q-1}, \Lambda_{q}\right)$, the following holds:

$$
\lim _{j \rightarrow+\infty}\left(j !\left(\Lambda_{q}-\ell_{j}^{(q)}\right)\right)^{1 / j}=\frac{b}{2}(\operatorname{Cap}(K))^{2} .
$$

(B) Assume $d \geq 1$. The counting function $N\left(\Lambda_{q-1}, \Lambda_{q}-\varepsilon, L_{\Omega, b}^{\tau}\right)$ has the asymptotics,

$$
N\left(\Lambda_{q-1}, \Lambda_{q}-\varepsilon, L_{\Omega, b}^{\tau}\right) \sim\left(\begin{array}{c}
q+d-1 \\
d-1
\end{array}\right) \frac{1}{d !}\left(\frac{|\log \varepsilon|}{\log |\log \varepsilon|}\right)^{d} \text { as } \quad \varepsilon \rightarrow 0^{+}
$$

Remark 1.3. The asymptotics in Equation (1.9) is weaker than that in Equation (1.8). It remains an open problem to finding an analogue for Equation (1.8) when $d>1$.

Remark 1.4. Theorems 1.1 and 1.2 were obtained for the Neumann case $(\tau \equiv 0)$ by the third author in Ref. 21, and our proofs will follow the same idea as in Ref. 21. However, in Ref. 21, the full details concerning the reduction to Toeplitz type operators were not written out explicitly. In this paper, we aim not only to generalize the Neumann result, but also to make the proof of Theorem 1.2 more transparent.

Remark 1.5. Our proof of Theorem 1.2 is carried out for $\tau$ being a self-adjoint pseudodifferential operator of order 0 on $\partial \Omega$. The proof can be generalized to self-adjoint pseudodifferential operators of order $t<1$. We also note that the pseudo-differential nature of the proof of 
Theorem 1.2 requires a fair amount of regularity on the boundary. Any considerable reduction of the regularity assumptions in Theorem 1.2 would require a new approach or a perturbation result for the left hand side of Equations (1.8) and (1.9).

The rest of the paper is devoted to the proof of Theorem 1.1 and Theorem 1.2. The proof of Theorem 1.1 is contained in Section II. The proof of Theorem 1.2 is divided into two sections: the bulk of the proof is contained in Section III except for a technical lemma, Lemma 3.14, which is proved in Section IV. Similarly to Refs. 21 and 22, the main idea in both proofs is to compare the resolvent of $L_{\Omega, b}^{\tau}$ with the resolvent of the Landau Hamiltonian. Roughly speaking, the resolvents are compact perturbation of one another.

Section II goes analogously to Ref. 21 [Section 3.1]. It is included for completeness and providing a notational introduction. As mentioned above, it relies heavily on the resolvent techniques introduced in Ref. 22, [Proposition 2.1].

The asymptotics in Theorem 1.2 comes from the spectral asymptotics of Toeplitz operators on the Landau levels; these deep results were proven in Refs. 9 and 20. We recall them in Subsection III A. The key step in obtaining the asymptotic accumulation of eigenvalues described in Theorem 1.2 is the reduction to the case of Toeplitz operators from Refs. 9 and 20 via a certain pseudo-differential operator on the boundary. The relevant pseudo-differential operators, e.g., boundary layer potentials and Dirichlet to Robin operators, are introduced in Subsections III B, III C, and III D. The reduction is carried out in Subsection III E apart from a technical lemma. Technical Lemma 3.14 states the equivalence of the quadratic form associated with a certain pseudo-differential operator of order 1 and the $H^{1 / 2}$-norm on the boundary. It is proven via standard pseudo-differential techniques in Section IV.

\section{PROOF OF THEOREM 1.1}

In this section, we prove Theorem 1.1. As remarked above, the proof goes along the lines of Ref. 21 [Section 3.1]. After adding on a Landau Hamiltonian $L_{K, b}^{-\tau}$ on $K$ (the sign of $-\tau$ comes from the orientation on the boundary), we can consider an operator densely defined in $L^{2}\left(\mathbb{R}^{2 d}\right)$ coinciding in form sense on the form domain of the usual Landau Hamiltonian. While $L_{K, b}^{-\tau}$ has a discrete spectrum, the proof of Theorem 1.1 is deduced below in Corollary 2.6 from the abstract results from Refs. 6 and 22 reviewed in Sec. II A.

\section{A. Two abstract results}

In this section, we state two abstract results. We will use the first result to conclude positivity of difference of resolvents and the second one to obtain the finiteness of eigenvalues above each Landau level.

Lemma 2.1 (Pushnitski-Rozenblum Ref. 22 [Proposition 2.1]). Assume that A and B are two self-adjoint positive operators satisfying the following hypotheses:

- $0 \notin \sigma(A) \cup \sigma(B)$.

- The form domain of $A$ contains that of B, i.e., $D\left(B^{1 / 2}\right) \subset D\left(A^{1 / 2}\right)$.

- For all $f \in D\left(B^{1 / 2}\right),\left\|A^{1 / 2} f\right\|=\left\|B^{1 / 2} f\right\|$, i.e., the quadratic forms of $A$ and $B$ agree on the form domain of $B$.

Then, $B^{-1} \leq A^{-1}$ in the quadratic form sense, i.e.,

$$
\left\langle B^{-1} f, f\right\rangle \leq\left\langle A^{-1} f, f\right\rangle \quad \forall f .
$$

Lemma 2.2 (Ref. 6, Theorem 9.4.7). Assume $A$ is a self-adjoint operator and $V$ a compact and positive operator such that the spectrum of $A$ in an interval $(\alpha, \beta)$ is discrete and does not accumulate at $\beta$. Then the spectrum of the operator $B=A+V$ in $(\alpha, \beta)$ is discrete and does not accumulate at $\beta$. 


\section{B. Some facts about the Landau Hamiltonian in $\mathbb{R}^{2 d}$}

In this section, we review classical results concerning the Landau Hamiltonian

$$
L=-\left(\nabla-i b \mathbf{A}_{0}\right)^{2} \quad \text { in } \mathbb{R}^{2 d} .
$$

Here $\mathbf{A}_{0}$ is the magnetic potential of a unit constant magnetic field of full rank introduced in (1.3), and $b$ is a positive constant. The form domain of $L$ is the magnetic Sobolev space

$$
H_{\mathbf{A}_{0}}^{1}\left(\mathbb{R}^{2 d}\right)=\left\{u \in L^{2}\left(\mathbb{R}^{2 d}\right):\left(\nabla-i b \mathbf{A}_{0}\right) u \in L^{2}\left(\mathbb{R}^{2 d}\right)\right\} \subseteq H_{\mathrm{loc}}^{1}\left(\mathbb{R}^{2 d}\right) .
$$

The spectrum of $L$ consists of infinitely degenerate eigenvalues called Landau levels,

$$
\sigma(L)=\sigma_{\text {ess }}(L)=\left\{\Lambda_{q}: q \in \mathbb{N} \backslash\{0\}\right\} .
$$

We denote by $\mathcal{L}_{q}$ the eigenspace associated with the Landau level $\Lambda_{q}$, i.e.,

$$
\mathcal{L}_{q}=\operatorname{Ker}\left(L-\Lambda_{q}\right) \quad \forall q \in \mathbb{N} \backslash\{0\} .
$$

We use the notation $P_{q}$ for the orthogonal projection onto the eigenspace $\mathcal{L}_{q}$.

The operator $L$ can be expressed in terms of creation and annihilation operators. We introduce the complex notation $z_{j}=x_{2 j-1}+i x_{2 j}, j=1, \ldots, d$, and let $\Psi=\frac{b}{4}|z|^{2}$ be a scalar potential for the magnetic field, i.e., $\Delta \Psi=b$. The differential expressions

$$
\bar{Q}_{j}=-2 i e^{-\Psi} \frac{\partial}{\partial z_{j}} e^{\Psi}, \quad Q_{j}=-2 i e^{\Psi} \frac{\partial}{\partial \bar{z}_{j}} e^{-\Psi}
$$

formally satisfy the following well known identities:

$$
\begin{aligned}
\bar{Q}_{j} & =Q_{j}^{*}, \quad 1 \leq j \leq d, \\
{\left[Q_{j}, \bar{Q}_{k}\right] } & =2 b \delta_{j k}, \quad 1 \leq j, k \leq d, \\
L & =\sum_{j=1}^{d} \bar{Q}_{j} Q_{j}+b d .
\end{aligned}
$$

\section{Extension of $L_{\Omega}$ to an operator in $L^{2}\left(\mathbb{R}^{2 d}\right)$}

We introduced the operator $L_{\Omega, b}^{\tau}$ with quadratic form $\mathfrak{I}_{\Omega, b}^{\tau}$ in (1.5). We will use also the corresponding operator in $K^{\circ}$, namely, $L_{K, b}^{-\tau}$. We will throughout the paper work under the assumption that the two quadratic forms $\mathfrak{l}_{\Omega, b}^{\tau}$ and $\mathfrak{I}_{K, b}^{-\tau}$ are strictly positive. This is always attainable after a shift of the quadratic forms by a constant. The effect of the constant is merely a shifting of the spectrums of all involved operators; hence we will for notational simplicity always assume that this constant is 0 .

Remark 2.3. Notice that, for $u \in H_{\mathbf{A}_{0}}^{1}\left(\mathbb{R}^{2 d}\right)$,

$$
\mathfrak{l}_{\Omega, b}^{\tau}\left(u_{\Omega}\right)+\mathfrak{l}_{K, b}^{-\tau}\left(u_{K}\right)=\int_{\mathbb{R}^{2 d}}\left|\left(\nabla-i b \mathbf{A}_{0}\right) u\right|^{2} d x .
$$

This motivates the usage of $-\tau$ for the quadratic form on $K$. For $u_{\Omega} \in D\left(L_{\Omega, b}^{\tau}\right)$ and $u_{K} \in D\left(L_{K, b}^{-\tau}\right)$,

$$
\partial_{R} u_{\Omega}=\partial_{R} u_{K}=0 \quad \text { on } \partial \Omega,
$$

where $\partial_{R}$ denotes the Robin differential expression from (1.4).

When there is no ambiguity, we will skip $b$ and $\tau$ from the notation and write $L_{\Omega}, L_{K}, \mathrm{l}_{\Omega}$, and $\mathrm{I}_{K}$ for the operators $L_{\Omega, b}^{\tau}, L_{K, b}^{-\tau}$, the quadratic forms $\mathfrak{I}_{\Omega, b}^{\tau}$ and $\mathfrak{l}_{K, b}^{-\tau}$, respectively.

Since $\Omega$ and $K$ are complementary in $\mathbb{R}^{2 d}$, the space $L^{2}\left(\mathbb{R}^{2 d}\right)$ is decomposed as a direct sum $L^{2}(\Omega) \oplus L^{2}(K)$. This permits us to extend the operator $L_{\Omega}$ in $L^{2}(\Omega)$ to an operator $\widetilde{L}$ in $L^{2}\left(\mathbb{R}^{2 d}\right)$. We let $\widetilde{L}=L_{\Omega} \oplus L_{K}$ in $D\left(L_{\Omega}\right) \oplus D\left(L_{K}\right) \subset L^{2}\left(\mathbb{R}^{2 d}\right)$. More precisely, $\widetilde{L}$ is the self-adjoint extension associated with the quadratic form

$$
\widetilde{\mathfrak{I}}(u)=\mathfrak{I}_{\Omega}\left(u_{\Omega}\right)+\mathfrak{I}_{K}\left(u_{K}\right), \quad u=u_{\Omega} \oplus u_{K} \in L^{2}\left(\mathbb{R}^{2 d}\right), \quad u_{\Omega} \in D\left(\mathfrak{I}_{\Omega}\right), \quad u_{K} \in D\left(\mathfrak{I}_{K}\right) .
$$


Since $\mathfrak{I}_{\Omega}$ and $\mathfrak{I}_{K}$ are strictly positive, we may speak of the inverse $\widetilde{L}^{-1}$ of $\widetilde{L}$. We have the following lemma.

Lemma 2.4. With $\widetilde{L}$ and $L_{\Omega}$ defined as above, we have the following:

(1) $\sigma_{\text {ess }}\left(L_{\Omega}\right)=\sigma_{\text {ess }}(\widetilde{L})$.

(2) $\lambda \in \sigma_{\text {ess }}\left(\widetilde{L}^{-1}\right) \backslash\{0\}$ if and only if $\lambda \neq 0$ and $\lambda^{-1} \in \sigma_{\text {ess }}\left(L_{\Omega}\right)$.

Proof. Since $\widetilde{L}=L_{\Omega} \oplus L_{K}$, then $\sigma(\widetilde{L})=\sigma\left(L_{\Omega}\right) \cup \sigma\left(L_{K}\right)$. But $K$ is compact and has a Lipschitz boundary, hence $L_{K}$ has a compact resolvent in Ref. 13 [Theorem 1.4.3.2]. Thus $\sigma_{\text {ess }}\left(L_{K}\right)=\varnothing$ and the first assertion in the lemma above follows. Moreover, $L_{\Omega}$ and $L_{K}$ are both strictly positive by the hypothesis, hence $0 \notin \sigma(\widetilde{L})$. It is straight forward that

$$
\sigma_{\mathrm{ess}}(\widetilde{L})=\left\{\lambda \in \mathbb{R} \backslash\{0\}: \lambda^{-1} \in \sigma_{\mathrm{ess}}\left(\widetilde{L}^{-1}\right)\right\} .
$$

\section{Essential spectrum of $L_{\Omega}$}

With the operator $\widetilde{L}$ introduced above, we can view $L_{\Omega}$ as a perturbation of the Landau Hamiltonian $L$ in $\mathbb{R}^{2 d}$ introduced in (2.1). Actually, we define $V: L^{2}\left(\mathbb{R}^{2 d}\right) \rightarrow L^{2}\left(\mathbb{R}^{2 d}\right)$ as

$$
V=\widetilde{L}^{-1}-L^{-1} \text {. }
$$

Then we have the following result on the operator $V$.

Lemma 2.5. The operator $V$ is positive and compact. Moreover, for all $f, g \in L^{2}\left(\mathbb{R}^{2 d}\right)$,

$$
\langle f, V g\rangle_{L^{2}\left(\mathbb{R}^{2 d}\right)}=\int_{\partial \Omega} \partial_{R} u \cdot \overline{\left(v_{\Omega}-v_{K}\right)} d S,
$$

where $u=L^{-1} f$ and $v=\widetilde{L}^{-1} g$.

Proof. Notice that the form domain $H_{\mathbf{A}_{0}}^{1}\left(\mathbb{R}^{2 d}\right)$ of $L$ is included in that of $\widetilde{L}$, and that for $u \in H_{\mathbf{A}_{0}}^{1}\left(\mathbb{R}^{2 d}\right)$, we have

$$
\widetilde{\mathfrak{I}}(u)=\int_{\mathbb{R}^{2 d}}\left|\left(\nabla-i b \mathbf{A}_{0}\right) u\right|^{2} d x .
$$

Invoking Lemma 2.1, we get that the operator $V$ is positive.

Let us establish the identity in (2.5). Set $f=L u$ and $g=\widetilde{L} v=L_{\Omega} v_{\Omega} \oplus L_{K} v_{K}$. Then

$$
\langle f, V g\rangle_{L^{2}\left(\mathbb{R}^{2} d\right)}=\int_{\Omega} L u \cdot \overline{v_{\Omega}} d x+\int_{K} L u \cdot \overline{v_{K}} d x-\int_{\Omega} u \cdot \overline{L_{\Omega} v_{\Omega}} d x-\int_{K} u \cdot \overline{L_{K} v_{K}} d x .
$$

The identity in (2.5) then follows by integration by parts and by using the boundary conditions $\partial_{R} v_{\Omega}=\partial_{R} v_{K}=0$.

Knowing that the zeroth order trace operators $H^{s_{1}}(K) \rightarrow H^{s_{2}}(\partial \Omega)$ and $H_{\mathrm{loc}}^{s_{1}}(\Omega) \rightarrow H^{s_{2}}(\partial \Omega)$ are compact whenever $s_{1}>s_{2}+1 / 2>1 / 2$, cf. Ref. 19 [Theorem 9.4, Chap. 1], we conclude from (2.5) that $V$ is a compact operator.

The localization of $V$ to the boundary carried out in Lemma 2.5 is by now a common triviality used in studying boundary value problems, but a sensation around the time of its invention by Birman, see more in Refs. 4 and 5. Theorem 1.1 follows as a corollary of Lemma 2.5.

Corollary 2.6. It holds that

$$
\sigma_{\text {ess }}\left(L_{\Omega}\right)=\left\{\Lambda_{q}: q \in \mathbb{N} \backslash\{0\}\right\},
$$

and for all $\varepsilon \in(0, b)$ and $q \in \mathbb{N} \backslash\{0\}$,

$$
\sigma\left(L_{\Omega}\right) \cap\left(\Lambda_{q}, \Lambda_{q}+\varepsilon\right) \text { is finite. }
$$


Proof. Invoking Lemma 2.4, it suffices to prove that

$$
\sigma_{\text {ess }}\left(\widetilde{L}^{-1}\right) \backslash\{0\}=\left\{\Lambda_{q}^{-1}: q \in \mathbb{N} \backslash\{0\}\right\}
$$

in order to get the result concerning the essential spectrum of $L_{\Omega}$. Notice that $\widetilde{L}^{-1}=L^{-1}+V$ with $V$ a compact operator. Hence by Weyl's theorem, $\sigma_{\text {ess }}\left(\widetilde{L}^{-1}\right)=\sigma_{\text {ess }}\left(L^{-1}\right)$. But we know from Section II B that $\sigma_{\text {ess }}\left(L^{-1}\right) \backslash\{0\}=\left\{\Lambda_{q}^{-1}: q \in \mathbb{N} \backslash\{0\}\right\}$ as was required to prove.

Since the operator $V$ is compact and positive, invoking Lemma 2.2, we get that $\sigma\left(\widetilde{L}^{-1}\right) \cap$ $\left(\Lambda_{q}^{-1}-\varepsilon, \Lambda_{q}^{-1}\right)$ is finite. This implies that $\sigma\left(L_{\Omega}\right) \cap\left(\Lambda_{q}, \Lambda_{q}+\varepsilon\right)$ is finite.

\section{PROOF OF THEOREM 1.2}

In this section, we prove Theorem 1.2. The main idea of the proof is, as mentioned above, to reduce the spectral asymptotics in (1.8) and (1.9) to a similar asymptotics for Toeplitz operators on the Landau levels from Refs. 9 and 20. The reduction to Toeplitz operators is by means of localizing to the boundary. The localization to the boundary is carried out using the identity (2.5).

\section{A. The spectrum of certain Toeplitz operators}

For $q \in \mathbb{N} \backslash\{0\}$ and a measurable set $U \subset \mathbb{R}^{2 d}$, the Toeplitz operator $S_{q}^{U}$ is defined by

$$
S_{q}^{U}=P_{q} \chi_{U} P_{q} \text { in } L^{2}\left(\mathbb{R}^{2 d}\right) .
$$

Here $\chi_{U}$ is the characteristic function of $U$. If $U$ is bounded, the theorem of Arzela-Ascoli implies that $\chi_{U} P_{q}$ is a compact operator, because Cauchy estimates for holomorphic functions can be generalized to the Landau levels. In particular, the Toeplitz operator $S_{q}^{U}$ is compact. We state the following deep results on these Toeplitz operators.

Theorem 3.1 (Ref. 9 [Lemma 3.2]). Assume that $U \subseteq \mathbb{R}^{2}$ is a bounded domain with a Lipschitz boundary. Given $q \in \mathbb{N} \backslash\{0\}$, denote by $s_{1}^{(q)} \geq s_{2}^{(q)} \geq \ldots$ the decreasing sequence of eigenvalues of $S_{q}^{U}$. Then,

$$
\lim _{j \rightarrow+\infty}\left(j ! s_{j}^{(q)}\right)^{1 / j}=\frac{b}{2}(\operatorname{Cap}(U))^{2} .
$$

Theorem 3.2 (Ref. 20 [Proposition 7.1]). Assume that $U \subseteq \mathbb{R}^{2 d}$ is a bounded domain. Given $q \in \mathbb{N}$, we let $n\left(\varepsilon, S_{q}^{U}\right)$ denote the number of eigenvalues of $S_{q}^{U}$ greater than $\varepsilon$. Then

$$
n\left(\varepsilon, S_{q}^{U}\right) \sim\left(\begin{array}{c}
q+d-1 \\
d-1
\end{array}\right) \frac{1}{d !}\left(\frac{|\log \varepsilon|}{\log |\log \varepsilon|}\right)^{d} \quad \text { as } \quad \varepsilon \rightarrow 0^{+} .
$$

The reader will recognize the structure of these results and notice that our main results look very much like them. Indeed, our main task will be to reduce our situation so that these results can be applied.

\section{B. The resolvent of the Landau Hamiltonian}

Since $L$ is strictly positive, $L^{-1}$ is a bounded operator in $L^{2}\left(\mathbb{R}^{2 d}\right)$ with range $D(L)$. Furthermore, $L^{-1}$ is an operator with an integral kernel that we denote by $G_{0}$. This integral kernel is well-known (see Ref. 25) to be

$$
G_{0}(z, \zeta)=\frac{2 b^{d-1}}{(4 \pi)^{d}} e^{b(\bar{z} \cdot \zeta-\bar{\zeta} \cdot z) / 4} I\left(\frac{b|z-\zeta|^{2}}{4}\right)
$$

where

$$
I(s)=\int_{0}^{+\infty} \frac{e^{-s \operatorname{coth}(t)}}{\sinh ^{d}(t)} d t
$$


Remark 3.3. The formula for $G_{0}(x, y)$ when $d=1$ is more commonly known,

$$
G_{0}(x, y)=\int_{0}^{+\infty} \frac{b}{2 \pi \sinh (b s)} \exp \left(\frac{i b}{2} x \wedge y-\frac{b}{4 \tanh (b s)}|x-y|^{2}\right) d s,
$$

where $x \wedge y=x_{1} y_{2}-x_{2} y_{1}=(\bar{z} \cdot \zeta-\bar{\zeta} \cdot z) / 2$ if $z=x_{1}+i x_{2}, \zeta=y_{1}+i y_{2}$

Lemma 3.4. $L^{-1}$ is an integral operator with kernel $G_{0}(z, \zeta)$ that has the following singularity at the diagonal $z=\zeta$ : there exist $a_{j} \in C^{\infty}\left(\mathbb{R}^{2 d} \times \mathbb{R}^{2 d}\right)$, for $j \in \mathbb{N} \backslash\{0\}$, and $b_{j} \in C^{\infty}\left(\mathbb{R}^{2 d} \times \mathbb{R}^{2 d}\right)$, for $j \in \mathbb{N} \backslash\{0\}$ with $j \geq \max (1, d-1)$, such that for any large enough $N$, there exists a function $\tilde{a}_{N+1} \in C^{N}\left(\mathbb{R}^{2 d} \times \mathbb{R}^{2 d}\right)$ such that

$$
\begin{aligned}
G_{0}(z, \zeta)= & \frac{1}{2 \pi} \log \left(\frac{1}{|z-\zeta|}\right)+\sum_{j=1}^{N} a_{j}(z, \zeta)|z-\zeta|^{2 j} \\
& +\sum_{j=1}^{N} b_{j}(z, \zeta)|z-\zeta|^{2 j} \log |z-\zeta|+\tilde{a}_{N}(z, z-\zeta), \quad d=1, \\
G_{0}(z, \zeta)= & \frac{\Gamma(d-1)}{2 \pi^{d}}|z-\zeta|^{2-2 d}+\sum_{j=1}^{N} a_{j}(z, \zeta)|z-\zeta|^{2-2 d+2 j} \\
& +\sum_{j=d-1}^{N} b_{j}(z, \zeta)|z-\zeta|^{2-2 d+2 j} \log |z-\zeta|+\tilde{a}_{N}(z, z-\zeta), \quad d>1 .
\end{aligned}
$$

The corresponding expansions, obtained by the term-wise differentiation, exist also for $\partial_{N} G_{0}(z, w)$ whenever $z, w \in \partial \Omega$. Moreover, $G_{0}(z, w)$ decays as a Gaussian as $|z-w| \rightarrow+\infty$ uniformly in both $z$ and $w$.

The proof of this lemma is of a computational nature and is deferred to the Appendix, where also the asymptotic expansion is computed explicitly.

Remark 3.5. For $d=1$, the integral (3.3) can be expressed in terms of the Whittaker function (see Ref. 8 [Section 4.9, formula (31)] and Ref. 7 [Chap. 6]) as

$$
\begin{aligned}
G_{0}(x, y)= & \frac{\pi^{3 / 2}}{b}\left(\frac{b}{8}|x-y|^{2}\right)^{-3 / 4} \exp \left(\frac{i b}{2} x \wedge y\right) \\
& \times\left[W_{\frac{1}{2},-\frac{1}{2}}\left(\frac{b}{2}|x-y|^{2}\right)+\frac{1}{2} W_{-\frac{1}{2},-\frac{1}{2}}\left(\frac{b}{2}|x-y|^{2}\right)\right] .
\end{aligned}
$$

Lemma 3.4 follows in this case from asymptotic formulae for Whittaker functions. ${ }^{7}$

\section{Boundary layer operators}

Recall that $K \subset \mathbb{R}^{2 d}$ has been assumed to be a compact subset of $\mathbb{R}^{2 d}$ with a smooth boundary and that we defined the domain $\Omega=\mathbb{R}^{2 d} \backslash K$. Since $\Omega$ and $K$ are complementary, the Hilbert space $L^{2}\left(\mathbb{R}^{2 d}\right)$ is naturally decomposed as the orthogonal direct sum $L^{2}(\Omega) \oplus L^{2}(K)$ in the sense that any function $u \in L^{2}\left(\mathbb{R}^{2 d}\right)$ can be uniquely represented as $u_{\Omega} \oplus u_{K}$, where $u_{\Omega}$ and $u_{K}$ are the restrictions of $u$ to $\Omega$ and $K$, respectively.

We let $\Psi^{*}(\partial \Omega)$ denote the filtered algebra of classical (also known under the name 1-step polyhomogeneous) pseudo-differential operators on the common boundary $\partial \Omega$ of $\Omega$ and $K$. For a reference on pseudo-differential operators, the reader is referred to Ref. 3 [Chap. 5], Ref. 15 [Chap. 18], or Ref. 24 [Chap. I]. We fix a classical pseudo-differential operator $\tau \in \Psi^{0}(\partial \Omega)$. The proofs also work for $\tau \in \Psi^{t}(\partial \Omega)$, for $t<1$, but become notationally more complicated. We restrict our attention to the case that $\tau$ is self-adjoint, in the current one and Sec. III D this assumption is needed merely to simplify proofs.

For the boundary considerations of this section, more care in the analysis of the Robin boundary differential expressions on $\partial \Omega$ defined in (1.4) is needed. We note that by Ref. 19 [Theorem 9.4, 
Chap. 1] the magnetic normal derivative, cf. (1.4), gives well defined continuous operators for $s \in \mathbb{R} \backslash\left\{\mathbb{Z}+\frac{1}{2}\right\}$ such that $s>3 / 2$,

$$
\partial_{N, \Omega}: H_{\mathrm{loc}}^{s}(\Omega) \rightarrow H^{s-3 / 2}(\partial \Omega) \quad \text { and } \quad \partial_{N, K}: H^{s}(K) \rightarrow H^{s-3 / 2}(\partial \Omega) .
$$

We remind the reader that the normal derivatives appearing in $\partial_{N, K}$ and $\partial_{N, \Omega}$ are both with respect to the unit outward normal vector to the boundary of $\Omega$. Again following Ref. 19 [Theorem 9.4, Chap. 1], the trace operators

$$
\gamma_{0, \Omega}: H_{\mathrm{loc}}^{s}(\Omega) \rightarrow H^{s-1 / 2}(\partial \Omega) \quad \text { and } \quad \gamma_{0, K}: H^{s}(K) \rightarrow H^{s-1 / 2}(\partial \Omega),
$$

mapping a function to their boundary value, are continuous for $s \in \mathbb{R} \backslash\left\{\mathbb{Z}+\frac{1}{2}\right\}$ such that $s>1 / 2$. For $s \in \mathbb{R} \backslash \mathbb{Z}+\frac{1}{2}$ such that $s>3 / 2$, we define the Robin boundary operators on $\partial \Omega$, following the expressions (1.4), by means of

$$
\begin{gathered}
\partial_{R, \Omega}:=\partial_{N, \Omega}+\tau \gamma_{0, \Omega}: H_{\mathrm{loc}}^{s}(\Omega) \rightarrow H^{s-3 / 2}(\partial \Omega) \quad \text { and } \\
\partial_{R, K}:=\partial_{N, K}+\tau \gamma_{0, K}: H^{s}(K) \rightarrow H^{s-3 / 2}(\partial \Omega) .
\end{gathered}
$$

As a rule, we suppress the $K$ and the $\Omega$ from the notation in these operators whenever the domain is clear from the context. We sometimes write $\left(\partial_{R}\right)_{x}$ in order to stress that the differentiation in (3.3) is with respect to the variable $x$.

With $G_{0}(x, y)$ as in (3.2), we define the operators $\mathcal{A}, \mathcal{B}, A$, and $B$, acting on functions defined on $\partial \Omega$, as

$$
\begin{aligned}
\mathcal{A} u(x) & =\int_{\partial \Omega} G_{0}(x, y) u(y) d S(y), \quad x \in \mathbb{R}^{2 d} \backslash \partial \Omega, \\
\mathcal{B} u(x) & =\int_{\partial \Omega}\left[\left(\partial_{N}\right)_{y} G_{0}(x, y)\right] u(y) d S(y), \quad x \in \mathbb{R}^{2 d} \backslash \partial \Omega, \\
A u(x) & =\int_{\partial \Omega} G_{0}(x, y) u(y) d S(y), \quad x \in \partial \Omega, \\
B u(x) & =\int_{\partial \Omega}\left[\left(\partial_{N}\right)_{y} G_{0}(x, y)\right] u(y) d S(y), \quad x \in \partial \Omega .
\end{aligned}
$$

The potentials $\mathcal{A}$ and $\mathcal{B}$ are usually called the single and double layer potentials. They satisfy $L \mathcal{A} u(x)=0$ and $L \mathcal{B} u(x)=0$ in $\mathbb{R}^{2 d} \backslash \partial \Omega$. We will write limit relations at the boundary for these potentials. We refer to Ref. 2 [Chap. 3, Section 12] where the corresponding potentials are considered for the Helmholtz operator, see also Ref. 3 [Section 5.7] for the low-dimensional case. Since, according to Lemma 3.4, the Green functions for both $L$ and the Helmholtz operator are globally estimated pseudo-differential operators with the same asymptotics in the leading terms as $x-y \rightarrow 0$, the limit relations in Ref. 3 apply here as well. For all $x_{0} \in \partial \Omega$, it holds that

$$
\begin{array}{ll}
\lim _{\substack{x \in K^{\circ} \\
x \rightarrow x_{0}}}(\mathcal{A} u)(x)=(A u)\left(x_{0}\right), \quad \lim _{\substack{x \in K^{\circ} \\
x \rightarrow x_{0}}}(\mathcal{B} u)(x)=\frac{1}{2} u\left(x_{0}\right)+(B u)\left(x_{0}\right), \\
\lim _{\substack{x \in \Omega \\
x \rightarrow x_{0}}}(\mathcal{A} u)(x)=(A u)\left(x_{0}\right), \quad \lim _{\substack{x \in \Omega \\
x \rightarrow x_{0}}}(\mathcal{B} u)(x)=-\frac{1}{2} u\left(x_{0}\right)+(B u)\left(x_{0}\right), \\
\lim _{\substack{x \in \Omega \\
x \rightarrow x_{0}}} \partial_{N}(\mathcal{A} u)(x)-\lim _{\substack{x \in K^{\circ} \\
x \rightarrow x_{0}}} \partial_{N}(\mathcal{A} u)(x)=u\left(x_{0}\right) .
\end{array}
$$

We recall from Refs. 2 and 3 that any function $u=u_{\Omega} \oplus u_{K} \in H_{\mathrm{loc}}^{s}(\Omega) \oplus H^{s}(K)$ exponentially decaying at $\infty$ and solving $L u=0$ in $\Omega \dot{\cup} K^{\circ}$ admits the representation by the formulas

$$
\begin{aligned}
& u=\mathcal{B}\left(\gamma_{0, K} u_{K}\right)-\mathcal{A}\left(\partial_{N, K} u_{K}\right), \quad \text { in } K^{\circ}, \\
& u=\mathcal{A}\left(\partial_{N, \Omega} u_{\Omega}\right)-\mathcal{B}\left(\gamma_{0, \Omega} u_{\Omega}\right), \quad \text { in } \Omega .
\end{aligned}
$$

Using the limit values (3.7), we obtain the following formulas connecting Dirichlet and Robin data at the boundary $\partial \Omega$ : 


$$
\begin{aligned}
& \left(B+A \tau-\frac{1}{2}\right) \gamma_{0, K} u_{K}=A\left(\partial_{R, K} u_{K}\right), \\
& \left(B+A \tau+\frac{1}{2}\right) \gamma_{0, \Omega} u_{\Omega}=A\left(\partial_{R, \Omega} u_{\Omega}\right) .
\end{aligned}
$$

We will use these relations to define the Dirichlet to Robin and Robin to Dirichlet operators in Section III D below. Before doing so, we present some results on the operators $A$ and $B$. Recall that for $p \geq 1$, the symmetrically normed ideal of weak Schatten class operators $\mathcal{J}_{p, w}\left(L^{2}(\partial \Omega)\right)$ consists of those compact operators $C$ on $L^{2}(\partial \Omega)$ whose singular values $\left\{\mu_{k}(C)\right\}_{k \in \mathbb{N}}$ behave like $\mu_{k}(C)=O\left(k^{-1 / p}\right)$ as $k \rightarrow \infty$. See more in Ref. 26 .

Lemma 3.6. The operators $A$ and $B$ are classical pseudo-differential operators of order -1 . Furthermore,

(1) A defines a self-adjoint operator on $L^{2}(\partial \Omega)$;

(2) A is elliptic with a constant principal symbol;

(3) A defines a Fredholm operator $A: L^{2}(\partial \Omega) \rightarrow H^{1}(\partial \Omega)$ whose index vanishes;

(4) $A$ and $B$, considered as operators on $L^{2}(\Omega)$, belong to the weak Schatten class $\mathcal{J}_{2 d-1, w}$ $\left(L^{2}(\partial \Omega)\right)$.

Proof. It follows directly from the asymptotics in Lemma 3.4 that $A$ and $B$ are classical pseudo-differential operators of order -1 , see for instance Ref. 15 [Theorem 18.2.8]. As such, the Weyl law on $\partial \Omega$ implies that $A$ and $B$ belong to the weak Schatten class $\mathcal{J}_{2 d-1, w}\left(L^{2}(\partial \Omega)\right)$. It is also clear from Lemma 3.4 that $\sigma_{-1}(A)=\frac{\Gamma(d-1)}{2 \pi^{d}}$. Hence $A$ is elliptic. Furthermore, Equation (3.2) implies that $G_{0}(z, w)=\overline{G_{0}(w, z)}$ so $A$ is self-adjoint on $L^{2}(\partial \Omega)$. Since $\sigma_{-1}(A)$ is a constant mapping, it is a lower order perturbation of an invertible pseudo-differential operator and the statement $\operatorname{ind}\left(A: L^{2}(\partial \Omega) \rightarrow H^{1}(\partial \Omega)\right)=0$ follows.

Lemma 3.7. The elliptic operator A defines an isomorphism $A: L^{2}(\partial \Omega) \rightarrow H^{1}(\partial \Omega)$.

Proof. To prove that $A$ is an isomorphism, we follow the proof of Ref. 27 [Chap. 7, Proposition 11.5] with the necessary modifications. Since the index of $A: L^{2}(\partial \Omega) \rightarrow H^{1}(\partial \Omega)$ vanishes, it suffices to prove that the operator $A$ is injective. By elliptic regularity, it suffices to prove that $A: C^{\infty}(\partial \Omega) \rightarrow C^{\infty}(\partial \Omega)$ is injective.

Assume that $h \in C^{\infty}(\partial \Omega)$ with $A h=0$. If we define $u \in C^{\infty}\left(K^{\circ}\right)$ by $u(x)=\mathcal{A} h(x), x \in K^{\circ}$, then $u$ satisfies

$$
\begin{cases}-\left(\nabla-i b \mathbf{A}_{0}\right)^{2} u=0 & \text { in } K^{\circ} \\ u=0 & \text { on } \partial \Omega\end{cases}
$$

We use (2.3) and integrate by parts, to get

$$
0=\left\langle-\left(\nabla-b \mathbf{A}_{0}\right)^{2} u, u\right\rangle_{L^{2}(K)}=b d\|u\|_{L^{2}(K)}^{2}+\sum_{j=1}^{d}\left\|Q_{j} u\right\|_{L^{2}(K)}^{2} .
$$

This implies that $u \equiv 0$ in $K$, i.e.,

$$
\mathcal{A} h(x) \equiv 0 \quad \text { in } K^{\circ} .
$$

It follows from the limit relations (3.7) that $\partial_{N}(\mathcal{A} h)(x)$ makes a jump across the boundary $\partial \Omega$ of size $h$, so if we let $w(x)=\mathcal{A} h(x), x \in \Omega$, then it satisfies

$$
\begin{cases}-\left(\nabla-i b \mathbf{A}_{0}\right)^{2} w=0 & \text { in } \Omega, \\ \partial_{N} w=h & \text { on } \partial \Omega .\end{cases}
$$

Since, again by (3.7), $\mathcal{A} h$ does not jump across $\partial \Omega$, we see by (3.9) that $w=0$ on $\partial \Omega$.

From the exponential decay of $G_{0}(x, y)$ as $|x-y| \rightarrow+\infty$, it follows that $w(x)=O\left(|x|^{-N}\right)$ as $|x| \rightarrow+\infty$ for all $N>0$. This also applies to all derivatives of $w$. Moreover $w$ is smooth. Hence we 
can integrate by parts in $\Omega$ to find

$$
0=\left\langle-\left(\nabla-i b \mathbf{A}_{0}\right)^{2} w, w\right\rangle_{L^{2}(\Omega)}=b d\|w\|_{L^{2}(\Omega)}^{2}+\sum_{j=1}^{d}\left\|Q_{j} w\right\|_{L^{2}(\Omega)}^{2},
$$

and hence $w \equiv 0$ in $\Omega$. From (3.10), we see that $h=0$ on $\partial \Omega$.

\section{The Dirichlet to Robin and Robin to Dirichlet operators}

Let $\varphi \in L^{2}(\partial \Omega)$ be given, and let $u$ be a solution with exponential decay at infinity to the exterior Robin problem

$$
\begin{cases}L u=0, & \text { in } \Omega, \\ \partial_{R} u=\varphi, & \text { on } \partial \Omega .\end{cases}
$$

We will see below in Equation (3.11) that the existence of $u$ is guaranteed in a subspace of finite codimension. This solution is unique, provided that certain orthogonality conditions are imposed. We denote by $T_{\Omega}^{R \rightarrow D} \varphi$ the boundary values of $u$ at $\partial \Omega$, whenever $\varphi$ admits a solution $u$. The operator $T_{\Omega}^{R \rightarrow D}: \varphi \mapsto T_{\Omega}^{R \rightarrow D} \varphi$ is called the exterior Robin to Dirichlet operator for the differential equation $L u=0$. We define the interior Robin to Dirichlet operator $T_{K}^{R \rightarrow D}$ in a similar way. Their inverse operators associating Robin data of solutions to their Dirichlet data are called the exterior and interior Dirichlet to Robin operators and are denoted by $T_{\Omega}^{D \rightarrow R}$ and $T_{K}^{D \rightarrow R}$, respectively.

Using the relations in (3.8), we find that these operators in fact are independent on the choice of solution $u$ for $\varphi$ outside a finite-dimensional subspace. It follows from (3.8) that

$$
\begin{aligned}
\left(B+A \tau-\frac{1}{2}\right) T_{K}^{R \rightarrow D} & =A, \quad \text { on } \quad A^{-1}\left(B+A \tau-\frac{1}{2}\right) C^{\infty}(\partial \Omega), \\
\left(B+A \tau+\frac{1}{2}\right) T_{\Omega}^{R \rightarrow D} & =A, \quad \text { on } \quad A^{-1}\left(B+A \tau+\frac{1}{2}\right) C^{\infty}(\partial \Omega), \\
T_{K}^{D \rightarrow R} & =A^{-1}\left(B+A \tau-\frac{1}{2}\right), \\
T_{\Omega}^{D \rightarrow R} & =A^{-1}\left(B+A \tau+\frac{1}{2}\right) .
\end{aligned}
$$

These equations determine $T_{K}^{R \rightarrow D}, T_{\Omega}^{R \rightarrow D}, T_{K}^{D \rightarrow R}$, and $T_{\Omega}^{D \rightarrow R}$ outside a finite-dimensional subspace while $B+A \tau \pm \frac{1}{2}$ are elliptic pseudo-differential operators of order 0. After a choice of extension, Equation (3.11) allows us to consider the operators $T_{K}^{R \rightarrow D}, T_{\Omega}^{R \rightarrow D}, T_{K}^{D \rightarrow R}$, and $T_{\Omega}^{D \rightarrow R}$ as pseudo-differential operators on $\partial \Omega$. More precisely, we have the following standard result for Dirichlet to Robin operators. The proof can be found in Ref. 27 [Appendix C of Chap. 12].

Proposition 3.8. The interior and exterior Robin to Dirichlet and Dirichlet to Robin operators are given by the relations in (3.11) and are elliptic pseudodifferential operators with constant principal symbols,

$$
T_{K}^{R \rightarrow D}, T_{\Omega}^{R \rightarrow D} \in \Psi^{-1}(\partial \Omega) \quad \text { and } \quad T_{K}^{D \rightarrow R}, T_{\Omega}^{D \rightarrow R} \in \Psi^{1}(\partial \Omega) .
$$

Very often, Equation (3.11) determines the Dirichlet to Robin operators; the operators $B+$ $A \tau \pm \frac{1}{2}$ are, in a sense made precise below, generically invertible. This fact is based on the following lemma.

Lemma 3.9. Assume that $M$ is a closed manifold and that $s>0$. Let $T \in \Psi^{0}(M)$ be an elliptic operator of index 0 and $A \in \Psi^{-s}(M)$ be an invertible operator $A: L^{2}(M) \rightarrow H^{s}(M)$. Viewing $A^{-1}$ and $A^{-1} T$ as unbounded operators on $L^{2}(M)$, then for any $\varepsilon \notin-\sigma\left(A^{-1} T\right)$, the bounded operator

$$
T+\varepsilon A: L^{2}(M) \rightarrow L^{2}(M)
$$

is invertible.

The statement of the lemma makes sense because $T$ is a pseudo-differential operator of order zero and as such it preserves the domain $H^{s}(M)$ of $A^{-1}$. We also note that by elliptic regularity, 
the set $-\sigma\left(A^{-1} T\right)$ is the same when changing the domain and range of $A^{-1} T$ to $H^{t}(M)$ for any $t$.

Proof. As $\operatorname{ind}(T)=0$ and $A$ is of negative order, the operator $T+\varepsilon A$ is an elliptic pseudodifferential operator of order 0 . As such, it defines an operator $H^{t}(M) \rightarrow H^{t}(M)$ with index 0 for any $\varepsilon$ and $t$. Hence $T+\varepsilon A$ is invertible if and only if $\operatorname{ker}(T+\varepsilon A)=0$. However, $A$ is invertible so $\operatorname{ker}(T+\varepsilon A)=0$ holds if and only if $\operatorname{ker}\left(A^{-1} T+\varepsilon\right)=0$. By elliptic regularity, these subspaces do not depend on the choice of domain in the Sobolev scale. The operator $A^{-1} T$ is an elliptic pseudo-differential operator of order $s$ with index 0 . It follows that $\operatorname{ind}\left(A^{-1} T+\varepsilon\right)=0$. Thus $\operatorname{ker}\left(A^{-1} T+\varepsilon\right)=0$ holds if and only if $-\varepsilon \notin \sigma\left(A^{-1} T\right)$.

We say that $\tau$ is generic if the operators

$$
T_{ \pm, \tau}:=B+A \tau \pm \frac{1}{2}
$$

are invertible. Note the following well-known consequence of elliptic regularity and the Fredholm property of elliptic operators, an elliptic pseudo-differential operator $D$ of order $m$ is invertible as an operator between Sobolev spaces $D: H^{s}(\partial \Omega) \rightarrow H^{s-m}(\partial \Omega)$, for some $s \in \mathbb{R}$, if and only if $D$ is invertible inside the algebra of pseudo-differential operators. The following corollary motivates the terminology generic.

Corollary 3.10. Letting $\tau, A$, and $B$ be as above. For all $\varepsilon \in[-1,1]$ outside a finite subset, $\tau+\varepsilon$ is generic.

Proof. By Ref. 24 [Theorem I.8.4], if the spectrum $\sigma\left(A^{-1} T_{ \pm, \tau}\right)$ is not equal to $\mathbb{C}$, it is a discrete subset of $\mathbb{C}$ and $[-1,1] \cap \sigma\left(-A^{-1} T_{+, \tau}\right) \cap \sigma\left(-A^{-1} T_{-, \tau}\right)$ is a finite set. The corollary follows from Lemma 3.9 provided that there exists $\lambda_{ \pm} \in \mathbb{C}$ such that $A^{-1} T_{ \pm, \tau}+\lambda_{ \pm}$are invertible. We note that the principal symbols $\pm \sigma_{1}\left(A^{-1} T_{ \pm, \tau}\right)$ are positive constant functions on $S^{*} \partial \Omega$. Existence of $\lambda_{ \pm} \in \mathbb{C}$ follows from the Gårding inequality Ref. 15 [Theorem 18.1.14]).

We turn our attention to associating Robin data on $\partial \Omega$ to Dirichlet data for functions in the Landau subspace $\mathcal{L}_{q}$ or more generally to solutions of the homogeneous equation $\left(L-\Lambda_{q}\right) u=0$ in $K^{\circ}$. The construction is well known and can be found in, for instance, Ref. 27 [Chap. 7.12 and Appendix C of Chap. 12].

Let $Q_{q}^{K} \subseteq C^{\infty}(\bar{K})$ denote the space of solutions $u \in C^{\infty}(\bar{K})$ to $\left(L-\Lambda_{q}\right) u=0$ in $K^{\circ}$ and let $Q_{q}^{\partial \Omega} \subseteq C^{\infty}(\partial \Omega)$ be the image of $Q_{q}^{K}$ under the restriction mapping $C^{\infty}(\bar{K}) \rightarrow C^{\infty}(\partial \Omega)$. Since $L-$ $\Lambda_{q}$ is a strongly elliptic operator in $K$, the space $Q_{q}^{\partial \Omega} \subseteq C^{\infty}(\partial \Omega)$ has finite codimension. Since the eigenvalue multiplicities of $L$ in $K$ equipped with Dirichlet conditions on $\partial \Omega$ are finite, the kernel of $Q_{q}^{K} \rightarrow Q_{q}^{\partial \Omega}$ is finite-dimensional.

This means that we can, for any function $\varphi \in Q_{q}^{\partial \Omega}$, solve

$$
\begin{cases}\left(L-\Lambda_{q}\right) u=0, & \text { in } K^{\circ}, \\ u=\varphi, & \text { on } \partial \Omega .\end{cases}
$$

The condition that $u$ is $L^{2}$-orthogonal to $\operatorname{ker}\left(Q_{q}^{K} \rightarrow Q_{q}^{\partial \Omega}\right)$ guarantees a unique solution. Define the corresponding solution operator

$$
{ }^{0} T_{q}^{D \rightarrow R}: Q_{q}^{\partial \Omega} \rightarrow C^{\infty}(\partial \Omega), \quad{ }^{0} T_{q}^{D \rightarrow R} \varphi:=\partial_{R, K} u .
$$

We let $T_{q}^{D \rightarrow R}: C^{\infty}(\partial \Omega) \rightarrow C^{\infty}(\partial \Omega)$ denote any extension of this operator, which exists since $Q_{q}^{\partial \Omega} \subseteq C^{\infty}(\partial \Omega)$ has finite codimension. The following result is standard and follows from Ref. 27 [Chap. 7].

Lemma 3.11. The operator $T_{q}^{D \rightarrow R}$ possesses the following properties.

(1) $T_{q}^{D \rightarrow R}$ is an elliptic pseudo-differential operator of order 1 with a constant positive principal symbol.

(2) For any $s, \operatorname{ind}\left(T_{q}^{D \rightarrow R}: H^{s}(\partial \Omega) \rightarrow H^{s-1}(\partial \Omega)\right)=0$. 
(3) There exists a number $c=c(\partial \Omega, q) \in \mathbb{R}$ such that $T_{q}^{D \rightarrow R}: H^{1 / 2}(\partial \Omega) \rightarrow H^{-1 / 2}(\partial \Omega)$ is invertible as an operator on $L^{2}(\partial \Omega)$ as long as $\tau \geq c$.

Proof. The computation of the principal symbol of $T_{q}^{D \rightarrow R}$ can be found in Proposition C.1 of Ref. 27 [Chap. 12]. The identity ind $\left(T_{q}^{D \rightarrow R}: H^{s}(\partial \Omega) \rightarrow H^{s-1}(\partial \Omega)\right)=0$ follows in the same way as in the proof of Lemma 3.6. From the index computation for $T_{q}^{D \rightarrow R}$, we conclude that $T_{q}^{D \rightarrow R}$ is an isomorphism if and only if $T_{q}^{D \rightarrow R}$ is injective. It follows from the Gårding inequality that there exists a positive constant $c$ such that for $\tau \geq 0$, for a certain constant $c_{\tau}^{\prime}>0$,

$$
\operatorname{Re}\left\langle T_{q}^{D \rightarrow R} u, u\right\rangle \geq c_{\tau}^{\prime}\|u\|_{H^{1 / 2}(\partial \Omega)}^{2}-c\|u\|_{L^{2}(\partial \Omega)}^{2} .
$$

Hence, as long as $\tau \geq c$, we have $\operatorname{Re}\left\langle T_{q}^{D \rightarrow R} u, u\right\rangle \geq c_{\tau-c}^{\prime}\|u\|_{H^{1 / 2}(\partial \Omega)}^{2}$. Injectivity of $T_{q}^{D \rightarrow R}: H^{1 / 2}(\partial \Omega)$ $\rightarrow H^{-1 / 2}(\partial \Omega)$ assuming $\tau \geq c$ follows.

Remark 3.12. We note that by construction, $T_{q}^{D \rightarrow R}$ coincides with $\partial_{R, K}$ outside a finitedimensional subspace. Hence, for $q \in \mathbb{N} \backslash\{0\}$, a finite rank smoothing operator $S_{q} \in \Psi^{-\infty}(\partial \Omega)$ exists, such that, as long as $u \in C^{\infty}(\bar{K})$ satisfies $\left(L-\Lambda_{q}\right) u=0$ in $K^{\circ}$, then

$$
\partial_{R, K} u=\left(T_{q}^{D \rightarrow R}+S_{q}\right) \gamma_{0, K} u \text {. }
$$

\section{E. Reduction to a Toeplitz operator}

In this subsection, we prove Theorem 1.2 modulo a technical lemma that we prove in Sec. IV. We assume as above that $K \subset \mathbb{R}^{2 d}$ is compact with a smooth boundary upon which $\tau$ is a classical pseudo-differential operator which we for simplicity assume to have order 0 . In Sec. III C, we made the assumption that $\tau$ was self-adjoint to simplify proofs while in this subsection it is necessary for the results to hold. Let $q \in \mathbb{N} \backslash\{0\}$ and pick $\delta>0$ such that

$$
\left(\left(\Lambda_{q}^{-1}-2 \delta, \Lambda_{q}^{-1}+2 \delta\right) \backslash\left\{\Lambda_{q}^{-1}\right\}\right) \cap \sigma_{\text {ess }}\left(\widetilde{L}^{-1}\right)=\varnothing .
$$

Denote by $\left\{r_{j}^{(q)}\right\}_{j \geq 1}$ the decreasing sequence of eigenvalues of $\widetilde{L}^{-1}$ in the interval $\left(\Lambda_{q}^{-1}, \Lambda_{q}^{-1}+\delta\right)$. For each $q \in \mathbb{N} \backslash\{0\}$, we introduce the operator

$$
T_{q}=P_{q} V P_{q},
$$

where, as before, $P_{q}$ is the orthogonal projection onto the eigenspace $\mathcal{L}_{q}$ associated with $\Lambda_{q}$ and $V=\widetilde{L}^{-1}-L^{-1}$. By Lemma $2.5, V$ is a positive and compact operator. These properties are inherited by $T_{q}$. Denote by $\left\{t_{j}^{(q)}\right\}$ the decreasing sequence of eigenvalues of $T_{q}$. The next lemma, proved in Ref. 22 [Proposition 2.2], shows that $r_{j}^{(q)}-\Lambda_{q}^{-1}$ are close to the eigenvalues of $T_{q}$.

Lemma 3.13 (Ref. 22, Proposition 2.2). Given $\varepsilon>0$ there exist integers $l$ and $j_{0}$ such that

$$
(1-\varepsilon) t_{j+l}^{(q)} \leq r_{j}^{(q)}-\Lambda_{q}^{-1} \leq(1+\varepsilon) t_{j-l}^{(q)}, \quad \forall j \geq j_{0} .
$$

The spectrum of $T_{q}$ will be related further to the spectrums of Toeplitz operators for generic operators $\tau$. Recall that given a compact domain $U \subset \mathbb{R}^{2 d}$, we introduced in (3.1) the Toeplitz operator $S_{q}^{U}$. We will prove now the following result.

Lemma 3.14. For all $q \in \mathbb{N} \backslash\{0\}$, there exists a finite-dimensional subspace $\mathcal{W}_{q} \subseteq \mathcal{L}_{q}$ such that if $K_{0} \subset K \subset K_{1}$ are compact domains with $\partial K_{i} \cap \partial K=\varnothing($ for $i=0$ and $i=1)$ there exists $a$ constant $C>1$ such that

$$
\frac{1}{C}\left\langle f, S_{q}^{K_{0}} f\right\rangle_{L^{2}\left(\mathbb{R}^{2 d}\right)} \leq\left\langle f, T_{q} f\right\rangle_{L^{2}\left(\mathbb{R}^{2 d}\right)} \leq C\left\langle f, S_{q}^{K_{1}} f\right\rangle_{L^{2}\left(\mathbb{R}^{2 d}\right)} \quad \forall f \in \mathcal{L}_{q} \ominus \mathcal{W}_{q} .
$$

The proof of Lemma 3.14 is by reduction of the operator $T_{q}$ to a pseudo-differential operator on the common boundary $\partial \Omega$ of $\Omega$ and $K$. We postpone the proof to Section IV below and continue instead with the proof of (1.8). 
Corollary 3.15. Whenever $K \subset \mathbb{R}^{2}$ is compact with $C^{\infty}$-boundary,

$$
\lim _{j \rightarrow+\infty}\left(j !\left(r_{j}^{(q)}-\Lambda_{q}^{-1}\right)\right)^{1 / j}=\frac{b}{2}(\operatorname{Cap}(K))^{2} .
$$

In particular, (1.8) holds true.

Proof. Invoking the variational min-max principle, the result of Lemma 3.14 provides us with a sufficiently large integer $j_{0} \in \mathbb{N}$ such that, for all $j \geq j_{0}$, we have,

$$
\frac{1}{C} s_{j, K_{0}}^{(q)} \leq t_{j}^{(q)} \leq C s_{j, K_{1}}^{(q)} .
$$

Here $\left\{s_{j, K_{0}}^{(q)}\right\}_{j}$ and $\left\{s_{j, K_{1}}^{(q)}\right\}_{j}$ are the decreasing sequences of eigenvalues of $S_{q}^{K_{0}}$ and $S_{q}^{K_{1}}$, respectively. Applying the result of Theorem 3.1 in the inequality above, we get

$$
\frac{b}{2}\left(\operatorname{Cap}\left(K_{0}\right)\right)^{2} \leq \lim _{j \rightarrow+\infty}\left(j ! t_{j}^{(q)}\right)^{1 / j} \leq \frac{b}{2}\left(\operatorname{Cap}\left(K_{1}\right)\right)^{2} .
$$

Since both $K_{0} \subset K \subset K_{1}$ are arbitrary, we get by making them close to $K$,

$$
\lim _{j \rightarrow+\infty}\left(j ! t_{j}^{(q)}\right)^{1 / j}=\frac{b}{2}(\operatorname{Cap}(K))^{2} .
$$

Applying the above asymptotic limit in the estimate of Lemma 3.13, we get the announced result in Corollary 3.15 above.

Corollary 3.16. Equation (1.9) holds true.

Proof. This is clear from Lemma 3.14 and Theorem 3.2.

Summing up the results of Corollaries 2.6, 3.15, and 3.16, we end up with the proof of Theorem 1.2. All that remains is to prove Lemma 3.14. That will be the subject of Sec. IV.

\section{PROOF OF LEMMA 3.14}

The aim of this section is to prove Lemma 3.14. The proof in this subsection goes along similar lines as in Subsection 4.2 of Ref. 21. Recall the operators $A$ and $B$ from (3.6).

Lemma 4.1. Consider the elliptic operators $T_{ \pm, \tau}=B+A \tau \pm \frac{1}{2} \in \Psi^{0}(\partial \Omega)$. There exist elliptic operators $R_{ \pm, \tau} \in \Psi^{0}(\partial \Omega)$, with principal symbol $\sigma_{0}\left(R_{ \pm, \tau}\right)= \pm 2$, such that the operators

$$
T_{ \pm, \tau} R_{ \pm, \tau}-1 \in \Psi^{-\infty}(\partial \Omega) \quad \text { and } \quad R_{ \pm, \tau} T_{ \pm, \tau}-1 \in \Psi^{-\infty}(\partial \Omega)
$$

are of finite rank.

Lemma 4.1 could be considered folklore. In lack of a reference, we provide a proof of its statement.

Proof. We let $\Psi_{\text {fin }}^{-\infty}(\partial \Omega) \subseteq \Psi^{0}(\partial \Omega)$ denote the ideal of finite rank smoothing operators. We consider the unital algebras

$$
\mathfrak{A}_{\text {fin }}:=\Psi \quad{ }^{0}(\partial \Omega) / \Psi_{\text {fin }}^{-\infty}(\partial \Omega) \text { an } \quad \mathrm{d} \mathfrak{A}:=\Psi^{0}(\partial \Omega) / \Psi^{-\infty}(\partial \Omega) .
$$

There is a quotient mapping $\mathfrak{A}_{\text {fin }} \rightarrow \mathfrak{A}$ whose kernel is $\Psi^{-\infty}(\partial \Omega) / \Psi_{\text {fin }}^{-\infty}(\partial \Omega)$. It follows by means of the standard techniques of pseudo-differential operators that an element $T \in \Psi^{0}(\partial \Omega)$ is elliptic if and only if the equivalence class $T \bmod \Psi^{-\infty}(\partial \Omega)$ is an invertible element of $\mathfrak{A}$. Hence the lemma follows if we can prove that if $\tilde{a} \in \mathfrak{A}_{\mathrm{fin}}$ satisfies that $a:=\tilde{a} \bmod \Psi^{-\infty}(\partial \Omega) / \Psi_{\text {fin }}^{-\infty}(\partial \Omega) \in \mathfrak{A}$ is invertible, then so is $\tilde{a}$.

We choose a lift $\tilde{r} \in \mathfrak{A}_{\text {fin }}$ of $a^{-1}$ and consider the elements in $\Psi^{-\infty}(\partial \Omega) / \Psi_{\text {fin }}^{-\infty}(\partial \Omega)$ defined by

$$
s_{L}:=\tilde{r} \tilde{a}-1_{\mathfrak{U}_{\mathrm{fin}}} \text { and } s_{R}:=\tilde{a} \tilde{r}-1_{\mathfrak{U}_{\mathrm{fin}}} .
$$


We can lift $s_{L}$ and $s_{R}$ to smoothing operators $S_{L}, S_{R} \in \Psi^{-\infty}(\partial \Omega)$. The operators $1+S_{L}$ and $1+S_{R}$ are elliptic operators of index 0 . Fredholm operators of index 0 are invertible modulo finite rank operators. If follows by elliptic regularity that $1+S_{L}$ and $1+S_{R}$ are invertible modulo finite rank smoothing operators. Hence $1_{\mathfrak{A}_{\text {fin }}}+s_{L}$ and $1_{\mathfrak{Q}_{\text {fin }}}+s_{R}$ are invertible elements of $\mathfrak{A}_{\text {fin }}$

Let us define $\tilde{r}_{R}:=\tilde{r}\left(1_{\mathfrak{U}_{\text {fin }}}+s_{R}\right)^{-1}$ and $\tilde{r}_{L}:=\left(1_{\mathfrak{U}_{\text {fin }}}+s_{L}\right)^{-1} \tilde{r}$. A direct computation shows that

$$
\tilde{a} \tilde{r}_{R}=\tilde{r}_{L} \tilde{a}=1_{\mathfrak{A}_{\text {fin }}} .
$$

Multiplying the identity $\tilde{a} \tilde{r}_{R}=1_{\mathfrak{l}_{\mathrm{fin}}}$ with $\tilde{r}_{L}$ from the left proves that $\tilde{r}_{R}=\tilde{r}_{L}$. It follows that $\tilde{a}$ is invertible with inverse $\tilde{r}_{L}$.

By Lemma 3.9, it is generically the case that $T_{ \pm, \tau}$ is invertible. To simplify notation we set $\hat{F}_{ \pm, \tau}:=R_{ \pm, \tau} T_{ \pm, \tau}-1$.

Lemma 4.2. Let $q \in \mathbb{N} \backslash\{0\}$. There exists a finite rank smoothing operator $F_{q}: L^{2}\left(\mathbb{R}^{2 d}\right) \rightarrow$ $L^{2}(\partial \Omega)$ such that for all $f, g \in L^{2}\left(\mathbb{R}^{2 d}\right)$,

$$
\left\langle f, T_{q} g\right\rangle_{L^{2}\left(\mathbb{R}^{2 d}\right)}=\frac{1}{\Lambda_{q}^{2}} \int_{\partial \Omega}\left(P_{q} f\right) \cdot \overline{T_{\tau, q}\left(P_{q} g\right)} d S+\left\langle\gamma_{0} P_{q} f, F_{q} g\right\rangle_{L^{2}(\partial \Omega)},
$$

where $T_{\tau, q}$ is the elliptic operator defined by

$$
T_{\tau, q}:=\left(T_{q}^{D \rightarrow R}+S_{q}\right)^{*}\left(R_{-, \tau}-R_{+, \tau}\right) A\left(T_{q}^{D \rightarrow R}+S_{q}\right) \in \Psi^{1}(\partial \Omega) .
$$

Proof. We set $u=L^{-1} P_{q} f=\Lambda_{q}^{-1} P_{q} f, v=\widetilde{L}^{-1} P_{q} g=v_{\Omega} \oplus v_{K}$, and $w=L^{-1} P_{q} g=\Lambda_{q}^{-1} P_{q} g$. Notice that

$$
\left\langle f, T_{q} g\right\rangle_{L^{2}\left(\mathbb{R}^{2 d}\right)}=\left\langle P_{q} f, V P_{q} g\right\rangle_{L^{2}\left(\mathbb{R}^{2 d}\right)}
$$

where $V$ is the operator defined in (2.5). Invoking Lemma 2.5, we write,

$$
\left\langle P_{q} f, V P_{q} g\right\rangle_{L^{2}\left(\mathbb{R}^{2 d}\right)}=\int_{\partial \Omega} \partial_{R} u \cdot \overline{\left(v_{\Omega}-v_{K}\right)} d S=\int_{\partial \Omega} \partial_{R} u \cdot \overline{\left(v_{\Omega}-w+w-v_{K}\right)} d S .
$$

Note that since $u \in H_{\mathbf{A}_{0}}^{1}\left(\mathbb{R}^{2 d}\right), \partial_{R} u=\partial_{R, K} u=\partial_{R, \Omega} u$. Using (3.11) and Lemma 4.1, we can write further,

$$
\begin{aligned}
\left\langle P_{q} f, V P_{q} g\right\rangle_{L^{2}\left(\mathbb{R}^{2 d}\right)}= & \int_{\partial \Omega} \partial_{R} u \cdot \overline{\left(R_{+, \tau} A\left(\partial_{R, \Omega}\left(v_{\Omega}-w\right)\right)+R_{-, \tau} A\left(\partial_{R, K}\left(w-v_{K}\right)\right)\right)} d S \\
& -\int_{\partial \Omega} \partial_{R} u \cdot \overline{\left.\hat{F}_{+, \tau}\left(v_{\Omega}-w\right)+\hat{F}_{-, \tau}\left(w-v_{K}\right)\right)} d S .
\end{aligned}
$$

Notice that $v_{\Omega}$ and $v_{K}$ are in the domain of the operators $L_{\Omega}$ and $L_{K}$, respectively, hence $\partial_{R} v_{\Omega}=$ $\partial_{R} v_{K}=0$. By construction, $L_{q} u=L_{q} w=0$ in $K$, and Remark 3.12 implies that

$$
\partial_{R} u=\left(T_{q}^{D \rightarrow R}+S_{q}\right) \gamma_{0} u \text { and } \partial_{R} w=\left(T_{q}^{D \rightarrow R}+S_{q}\right) \gamma_{0} w,
$$

for some finite rank smoothing operator $S_{q} \in \Psi^{-\infty}(\partial \Omega)$. Consequently, after applying this identity to the first term in (4.2), we get

$$
\begin{aligned}
\left\langle P_{q} f, V P_{q} g\right\rangle_{L^{2}\left(\mathbb{R}^{2 d}\right)}+\int_{\partial \Omega} \partial_{R} u \cdot \overline{\left.\hat{F}_{+, \tau}\left(v_{\Omega}-w\right)+\hat{F}_{-, \tau}\left(w-v_{K}\right)\right)} d S \\
=\int_{\partial \Omega}\left(T_{q}^{D \rightarrow R}+S_{q}\right) u \cdot \overline{\left(R_{-, \tau}-R_{+, \tau}\right) A\left(T_{q}^{D \rightarrow R}+S_{q}\right) w} d S=\left\langle u, T_{\tau, q} w\right\rangle_{L^{2}(\partial \Omega)} .
\end{aligned}
$$

As for the second term in (4.2),

$$
\begin{aligned}
\int_{\partial \Omega} \partial_{R} u \cdot \overline{\left.\hat{F}_{+, \tau}\left(v_{\Omega}-w\right)+\hat{F}_{-, \tau}\left(w-v_{K}\right)\right)} d S \\
\quad=\int_{\partial \Omega} u \cdot \underbrace{\overline{\left(T_{q}^{D \rightarrow R}+S_{q}\right)^{*}\left(\Lambda_{q}^{-1}\left(\hat{F}_{-, \tau}-\hat{F}_{+, \tau}\right)+\left(\hat{F}_{+, \tau} L_{\Omega}^{-1}-\hat{F}_{-, \tau} L_{K}^{-1}\right)\right) P_{q} g}}_{\Lambda_{q} F_{q}} d S .
\end{aligned}
$$

Since $\hat{F}_{ \pm, \tau}$ are of finite rank, it is clear that $F_{q}$ is of finite rank. 
Lemma 4.3. The operator $T_{\tau, q}$ has a discrete spectrum, and there exists a finite rank operator $S_{\tau, q} \in \Psi^{-\infty}(\partial \Omega)$ such that for some $b, C>0$,

$$
\|\varphi\|_{H^{1 / 2}(\partial \Omega)} \leq C\left(\operatorname{Re}\left\langle\varphi, T_{\tau, q} \varphi\right\rangle_{L^{2}(\partial \Omega)}+b\left\|S_{\tau, q} \varphi\right\|_{L^{2}(\partial \Omega)}\right) .
$$

Proof. By an argument similar to that of Corollary 3.10, it follows from Ref. 24 [Theorem I.8.4] and the Gårding inequality that $T_{\tau, q}$ has a discrete spectrum. We define the elliptic self-adjoint first order pseudo-differential operator

$$
\tilde{T}_{\tau, q}:=\frac{1}{2}\left(T_{\tau, q}+T_{\tau, q}^{*}\right) .
$$

Since $T_{\tau, q}$ has a positive principal symbol, we see that $T_{\tau, q}-\tilde{T}_{\tau, q} \in \Psi^{0}(\partial \Omega)$ defines a bounded operator. The Gårding inequality implies that for some $b, C>0$,

$$
\|\varphi\|_{H^{1 / 2}(\partial \Omega)} \leq C\left(\left\langle\varphi, \tilde{T}_{\tau, q} \varphi\right\rangle_{L^{2}(\partial \Omega)}+b\|\varphi\|_{L^{2}(\partial \Omega)}\right) .
$$

Since $\tilde{T}_{\tau, q}$ is of order 1, elliptic, and self-adjoint, its spectrum is a discrete subset of $\mathbb{R}$. The Gårding inequality implies that $\tilde{T}_{\tau, q}$ is bounded from below, so the spectrum of $\tilde{T}_{\tau, q}$ only accumulates at $+\infty$ and there are only finitely many non-positive eigenvalues. We define $S_{\tau, q} \in \Psi^{-\infty}(\partial \Omega)$ as the finite rank projection onto the non-positive eigenspace of $\tilde{T}_{\tau, q}$. For a, possibly new, constant $C$, the lemma follows because

$$
\operatorname{Re}\left\langle\varphi, T_{\tau, q} \varphi\right\rangle_{L^{2}(\partial \Omega)}=\left\langle\varphi, \tilde{T}_{\tau, q} \varphi\right\rangle_{L^{2}(\partial \Omega)}
$$

\section{Proposition 4.4. The linear operator}

$$
H^{1}(K) \rightarrow H^{-1}(K) \oplus H^{1 / 2}(\partial \Omega), \quad f \mapsto\left(L-\Lambda_{q}\right) f \oplus \gamma_{0, K} f,
$$

is Fredholm.

Proof. We note that the strongly elliptic differential operator $L-\Lambda_{q}$ defines an elliptic boundary value problem when equipped with the Dirichlet condition as in (4.4). It follows that the linear operator given in (4.4) is Fredholm because it is a compact perturbation of the operator $f \mapsto(L+\lambda) f \oplus \gamma_{0, K} f$ which is invertible for $\mathfrak{R}(\lambda)$ large enough.

Remark 4.5. As a consequence of Proposition 4.4, there exists a bounded linear extension mapping $\mathfrak{Q}_{q}: H^{1 / 2}(\partial \Omega) \rightarrow H^{1}(K)$ satisfying $\gamma_{0} \mathfrak{Q}_{q} \varphi=\varphi$ and $\left(L-\Lambda_{q}\right) \mathfrak{Q}_{q} \varphi=0$ in $K^{\circ}$ for $\varphi$ outside a finite-dimensional subspace of $H^{1 / 2}(\partial \Omega)$. This finite-dimensional subspace can be chosen as the orthogonal complement of the space $\gamma_{0, K}\left(\operatorname{ker}\left(\left(L-\Lambda_{q}\right): H^{1}(K) \rightarrow H^{-1}(K)\right)\right)$. We conclude that there exists a finite rank operator $F_{K}$ on $H^{1}(K)$, smoothing in the interior, such that whenever $f \in H^{1}(K)$ satisfies $\left(L-\Lambda_{q}\right) f=0$ in $K^{\circ}$,

$$
\|f\|_{H^{1}(K)} \leq C\left(\left\|\gamma_{0} f\right\|_{H^{1 / 2}(\partial \Omega)}+\left\|F_{K} f\right\|_{H^{1}(K)}\right),
$$

for some constant $C$.

Lemma 4.6. Let $q \in \mathbb{N} \backslash\{0\}$ and let $F_{q}: L^{2}\left(\mathbb{R}^{2 d}\right) \rightarrow L^{2}(\partial \Omega)$ be the finite rank operator of Lemma 4.2, $S_{\tau, q} \in \Psi^{-\infty}(\partial \Omega)$ the finite rank operator of Lemma 4.3 and $P_{q}$ the orthogonal projection on the Landau level $\mathcal{L}_{q}$. There exist constants $b>0, C>1$, such that for all $f \in L^{2}\left(\mathbb{R}^{2 d}\right)$, it holds that

$$
\begin{aligned}
& \frac{1}{C}\left\|\gamma_{0} P_{q} f\right\|_{H^{1 / 2}(\partial \Omega)}\left(\left\|\gamma_{0} P_{q} f\right\|_{H^{1 / 2}(\partial \Omega)}-\left\|F_{q} f\right\|_{L^{2}(\partial \Omega)}-b\left\|S_{\tau, q} \gamma_{0} P_{q} f\right\|_{L^{2}(\partial \Omega)}\right) \\
& \quad \leq\left\langle f, T_{q} f\right\rangle_{L^{2}\left(\mathbb{R}^{2} d\right)} \leq C\left\|\gamma_{0} P_{q} f\right\|_{H^{1 / 2}(\partial \Omega)}\left(\left\|\gamma_{0} P_{q} f\right\|_{H^{1 / 2}(\partial \Omega)}+\left\|F_{q} f\right\|_{L^{2}(\partial \Omega)}\right) .
\end{aligned}
$$

Proof. Lemma 3.6 states that the operator $T_{\tau, q}$ from Lemma 4.2 is an elliptic pseudo-differential operator of order 1 with a positive principal symbol. Hence, by Lemma 4.3, there are constants $b, C>1$ such that

$$
\frac{1}{C}\|\varphi\|_{H^{1 / 2}(\partial \Omega)}\left(\|\varphi\|_{H^{1 / 2}(\partial \Omega)}-b\left\|S_{\tau, q} \varphi\right\|_{L^{2}(\partial \Omega)}\right) \leq \operatorname{Re}\left\langle\varphi, T_{\tau, q} \varphi\right\rangle_{L^{2}(\partial \Omega)} \leq C\|\varphi\|_{H^{1 / 2}(\partial \Omega)}^{2},
$$


for all $\varphi \in H^{1 / 2}(\partial \Omega)$. Applying the above estimates with $\varphi=\gamma_{0} P_{q} f$ and $f \in L^{2}\left(\mathbb{R}^{2 d}\right)$ and recalling (4.1), we get that the double inequality announced in the above lemma holds for all $f \in L^{2}\left(\mathbb{R}^{2 d}\right)$ due to Lemma 4.2 and the fact that

$$
\operatorname{Re}\left\langle\varphi, T_{\tau, q} \varphi\right\rangle_{L^{2}(\partial \Omega)}=\left\langle f, T_{q} f\right\rangle+\operatorname{Re}\left\langle\varphi, F_{q} f\right\rangle_{L^{2}(\partial \Omega)} .
$$

Proof of Lemma 3.14. Let $S_{\tau, q} \in \Psi^{-\infty}(\partial \Omega)$ denote the finite rank operator from Lemma 4.2. The operator $S_{\tau, q} \gamma_{0} P_{q}: L^{2}\left(\mathbb{R}^{2 d}\right) \rightarrow L^{2}(\partial \Omega)$ is a well defined finite rank operator since $S_{\tau, q}$ is finite rank and $P_{q} L^{2}\left(\mathbb{R}^{2 d}\right) \subseteq C^{\infty}\left(\mathbb{R}^{2 d}\right)$. Recall the finite rank operator $F_{K}$ on $H^{1}(K)$ from Remark 4.5 and let $F_{K} P_{q}: L^{2}\left(\mathbb{R}^{2 d}\right) \rightarrow H^{1}(K)$ denote the finite rank operator $\left.f \mapsto\left(P_{q} f\right)\right|_{K} \mapsto F_{K}\left[\left.\left(P_{q} f\right)\right|_{K}\right]$ which is well defined since $\left.\left(P_{q} f\right)\right|_{K} \in C^{\infty}(\bar{K})$. We define the space $\mathcal{W}_{q} \subseteq \mathcal{L}_{q}$ by means of

$$
\mathcal{L}_{q} \ominus \mathcal{W}_{q}:=\operatorname{ker}\left(S_{\tau, q} \gamma_{0} P_{q}\right) \cap \operatorname{ker} F_{q} \cap \operatorname{ker} F_{K} P_{q} \cap \mathcal{L}_{q} \subseteq \mathcal{L}_{q} .
$$

The space $\mathcal{W}_{q}$ is of finite dimension because all operators $S_{\tau, q} \gamma_{0} P_{q}, F_{q}$, and $F_{K} P_{q}$ are of finite rank.

Step 1. Lower bound.

We prove that the lower bound in (3.13) is valid for all $f \in \mathcal{W}_{q}^{\perp}$. For simplicity, we set $\varphi:=\gamma_{0} f$. By the definition of $T_{q}$ from (3.12), the estimate of Lemma 4.6 gives

$$
\left\langle f, T_{q} f\right\rangle_{L^{2}\left(\mathbb{R}^{2 d}\right)} \geq \frac{1}{C}\|\varphi\|_{H^{1 / 2}(\partial \Omega)}^{2} .
$$

So it suffices to prove that

$$
\left\langle f, S_{q}^{K} f\right\rangle_{L^{2}\left(\mathbb{R}^{2 d}\right)} \leq C^{\prime}\|\varphi\|_{H^{1 / 2}(\partial \Omega)}^{2},
$$

for some positive constant $C^{\prime}$. Recalling the definition of $S_{q}^{K}$, and using that $\|f\|_{L^{2}(K)} \leq\|f\|_{H^{1}(K)}$, this follows once showing the estimate

$$
\|f\|_{H^{1}(K)} \leq C^{\prime}\|\varphi\|_{H^{1 / 2}(\partial \Omega)} .
$$

Since $\left(L-\Lambda_{q}\right) f=0$, this estimate follows from Remark 4.5.

Step 2. Upper bound.

Now we establish the upper bound in (3.13). Let $f \in L^{2}\left(\mathbb{R}^{2 d}\right)$ and $u=P_{q} f$, the projection of $f$ onto the eigenspace $\mathcal{L}_{q}$. Notice that the trace theorem, Ref. 19 [Theorem 9.4, Chap. 1] gives

$$
\left\|\gamma_{0} u\right\|_{H^{1 / 2}(\partial \Omega)} \leq C\|u\|_{H^{2}(K)},
$$

for some positive constant $C$. Notice that $\left(L-\Lambda_{q}\right) u=0$. By the elliptic regularity, given a domain $K_{1}$ such that $K \subset K_{1}$, there exists a constant $C_{K_{1}}$ such that

$$
\|u\|_{H^{2}(K)} \leq C_{K_{1}}\left(\left\|L_{q} u\right\|_{L^{2}\left(K_{1}\right)}+\|u\|_{L^{2}\left(K_{1}\right)}\right)=C_{K_{1}}\|u\|_{L^{2}\left(K_{1}\right)} .
$$

Summing up, we get

$$
\left\|\gamma_{0} P_{q} f\right\|_{H^{1 / 2}(\partial \Omega)} \leq C\left\|P_{q} f\right\|_{L^{2}\left(K_{1}\right)}, \quad \forall f \in L^{2}\left(\mathbb{R}^{2 d}\right) .
$$

Substituting the above inequality in the estimate of Lemma 4.6, we obtain the upper bound announced in (3.13).

\section{ACKNOWLEDGMENTS}

The authors wish to thank Grigori Rozenblum who suggested the approach we used from the theory of pseudo-differential operators. Part of this work has been prepared in the Erwin Schrödinger Institute (ESI)-Vienna which is gratefully acknowledged. The authors M.G. and M.P.S. also wish to thank the Mittag-Leffler institute for a productive stay in 2012. AK is supported by a grant from Lebanese University.

\section{APPENDIX: PROOF OF LEMMA 3.4}

The proof of Lemma 3.4 and the expansion of the function $G_{0}$ defined in (3.2) are based on an expansion of the function $I$. 
Lemma A.1. The function

$$
I(s):=\int_{0}^{+\infty} \frac{e^{-s \operatorname{coth}(t)}}{\sinh ^{d}(t)} d t
$$

can be written as

$$
I(s)=I_{0}(s)+I_{\infty}(s)
$$

where

(1) $I_{0}(s), I_{\infty}(s)=O\left(e^{-s}\right)$ as $s \rightarrow+\infty$.

(2) $I_{\infty} \in C^{\infty}(\mathbb{R})$.

(3) The function $I_{0} \in C^{\infty}\left(\mathbb{R}_{+}\right)$admits an asymptotic expansion for small $s$,

$$
I_{0}(s)=\sum_{j=1-d}^{+\infty}\left(e^{-s a} c_{j}-c_{j}^{\prime}\right) s^{j}-\sum_{j=0}^{+\infty} d_{j} s^{j} \log (s),
$$

where

$$
\begin{aligned}
& c_{j}:= \begin{cases}\sum_{k=0}^{\lfloor(d-j) / 2\rfloor-1}(-1)^{k}\left(\begin{array}{c}
\frac{d-2}{2} \\
k
\end{array}\right)(d-2(k+1))_{j+1} a^{d-2 k-1-j}, & 1-d \leq j<0, \\
\sum_{k=\lceil(d-j-1) / 2\rceil-1}^{+\infty}(-1)^{k}\left(\begin{array}{c}
\frac{d-2}{2} \\
k
\end{array}\right) \frac{a^{d-2 k-1+j}}{(2 k+1-d)_{j+1}}, & 0 \leq j \leq d-2, \\
0, & j>d-2,\end{cases} \\
& \left(\sum_{\substack{j \geq k \geq 1 \\
j-k-d=1 \bmod 2}} \frac{(-1)^{(j+k+d-1) / 2} a^{k}}{(j-k) ! k \cdot k !}\left(\begin{array}{c}
\frac{d-2}{2} \\
\frac{j-k+d-1}{2}
\end{array}\right)\right. \\
& +\frac{(-1)^{(j+d-1) / 2}}{j !}\left(\begin{array}{c}
\frac{d-2}{2} \\
\frac{j+d-1}{2}
\end{array}\right)(\gamma+\log (a)+1), \quad j-d \equiv 1 \bmod 2, j \geq 1, \\
& c_{j}^{\prime}:=\left\{\sum_{\substack{j \geq k \geq 1 \\
j-k-d \equiv 1 \bmod 2}} \frac{(-1)^{(j+k+d-1) / 2} a^{k}}{(j-k) ! k \cdot k !}\left(\begin{array}{c}
\frac{d-2}{2} \\
\frac{j-k+d-1}{2}
\end{array}\right), \quad j-d \equiv 0 \bmod 2, j \geq 1,\right. \\
& \gamma+\log (a)+1, \quad j=0 \text { and } d \text { is odd, } \\
& 0 \text {, } \\
& d_{j}:=\sum_{\substack{j \geq k \geq 1 \\
j-k-d=1 \bmod 2}} \frac{(-1)^{(j+k+d-1) / 2} a^{k}}{(j-k) ! k \cdot k !}\left(\begin{array}{c}
\frac{d-2}{2} \\
\frac{j-k+d-1}{2}
\end{array}\right) . \\
& j=0 \text { and } d \text { is even, }
\end{aligned}
$$

Here $a=\operatorname{coth}(1)$ and $\gamma$ is the Euler-Mascheroni constant.

Proof. We write

$$
I_{0}(s):=\int_{0}^{1} \frac{e^{-s \operatorname{coth}(t)}}{\sinh ^{d}(t)} d t \quad \text { and } \quad I_{\infty}(s):=\int_{1}^{+\infty} \frac{e^{-s \operatorname{coth}(t)}}{\sinh ^{d}(t)} d t .
$$

It is easily verified that $I_{\infty} \in C^{\infty}(\mathbb{R})$. Since $\operatorname{coth}(t) \geq 1$ for $t \geq 1, I_{\infty}(s)=O\left(e^{-s}\right)$ as $s \rightarrow+\infty$. Since $\operatorname{coth}(t) \geq 6 / 5$ for $t \in[0,1], I_{0}(s)=O\left(e^{-s}\right)$ as $s \rightarrow+\infty$ follows once one notices that the singularity at $t=0$ from the $\sinh ^{d}(t)$ is canceled by $e^{-s \operatorname{coth}(t) / 6}$.

Let us turn to the unpleasant computation of $I_{0}$. After the change of variables $u=\operatorname{coth}(t)$, the integral defining $I_{0}$ transforms to

$$
I_{0}(s)=\int_{a}^{+\infty} e^{-s u}\left(u^{2}-1\right)^{\frac{d-2}{2}} d u=\int_{a}^{+\infty} e^{-s u} u^{d-2}\left(1-\frac{1}{u^{2}}\right)^{\frac{d-2}{2}} d u .
$$


Here $a:=\operatorname{coth}(1)>1$. Using the Taylor expansion of $\left(1-\frac{1}{u^{2}}\right)^{\frac{d-2}{2}}$, which converges uniformly for $u \geq \operatorname{coth}(1)$, we arrive at the identity

$$
\begin{aligned}
I_{0}(s) & =\sum_{k=0}^{+\infty}(-1)^{k}\left(\begin{array}{c}
\frac{d-2}{2} \\
k
\end{array}\right) \int_{a}^{+\infty} e^{-s u} u^{d-2(k+1)} d u \\
& =\sum_{k=0}^{+\infty}(-1)^{k}\left(\begin{array}{c}
\frac{d-2}{2} \\
k
\end{array}\right) s^{2 k+1-d} g_{d-2(k+1)}(s a),
\end{aligned}
$$

where

$$
g_{m}(t):=\int_{t}^{+\infty} e^{-u} u^{m} d u .
$$

After an integration by parts, one arrives at the identity

$$
g_{m}(t)= \begin{cases}e^{-t} \sum_{j=0}^{m}(m)_{j+1} t^{m-j}, & m \geq 0, \\ e^{-t} \sum_{j=1}^{-m-1} \frac{t^{m+j}}{(-m-1)_{j}}-\frac{\gamma+\log (t)+\sum_{j=0}^{+\infty}(-1)^{j} \frac{t^{j}}{j \cdot j !}}{(-m-1) !}, & m<0 .\end{cases}
$$

Here $(m)_{l}:=m(m-1) \cdots(m-l+1)$ denotes the Pochhammer symbol. Putting this into (A2),

$$
\begin{aligned}
I_{0}(s)= & \sum_{k=0}^{\lfloor(d-2) / 2\rfloor} \sum_{j=0}^{d-2(k+1)}(-1)^{k}\left(\begin{array}{c}
\frac{d-2}{2} \\
k
\end{array}\right)(d-2(k+1))_{j} e^{-s a} a^{d-2(k+1)-j} s^{-j-1} \\
& +\sum_{k=\lfloor d / 2\rfloor}^{+\infty}(-1)^{k}\left(\begin{array}{c}
\frac{d-2}{2} \\
k
\end{array}\right) e^{-s a} \sum_{j=1}^{2(k+1)-d} \frac{a^{d-2(k+1)-j}}{(2 k+1-d)_{j}} s^{j-1} \\
& -\sum_{k=\lfloor d / 2\rfloor}^{+\infty}(-1)^{k}\left(\begin{array}{c}
\frac{d-2}{2} \\
k
\end{array}\right) \frac{s^{2 k+1-d}(\gamma+\log (a)+1)+s^{2 k+1-d} \log (s)}{(2 k+1-d) !} \\
& -\sum_{k=\lfloor d / 2\rfloor}^{+\infty} \sum_{j=1}^{+\infty}(-1)^{k+j}\left(\begin{array}{c}
\frac{d-2}{2} \\
k
\end{array}\right) \frac{a^{j}}{j \cdot j !} \frac{s^{2 k+1-d+j}}{(2 k+1-d) !} .
\end{aligned}
$$

Rearranging these terms leads to the expression (A1).

Proof of Lemma 3.4. Using Lemma A.1, we have that

$$
\begin{aligned}
G_{0}(z, \zeta)=\sum_{j=1-d}^{+\infty} & \frac{b^{j+d-1}}{2^{2 d+2 j-1} \pi^{d}} e^{b(\bar{z} \cdot \zeta-\bar{\zeta} \cdot z) / 4}\left(e^{-a b|z-\zeta|^{2} / 4} c_{j}-c_{j}^{\prime}\right)|z-\zeta|^{2 j} \\
& -\sum_{j=0}^{+\infty} d_{j} \frac{b^{j+d-1}}{2^{2 d+2 j-1} \pi^{d}} e^{b(\bar{z} \cdot \zeta-\bar{\zeta} \cdot z) / 4}|z-\zeta|^{2 j} \log \left(\frac{b|z-\zeta|^{2}}{4}\right) \\
& +\frac{2 b^{d-1}}{(4 \pi)^{d}} e^{b(\bar{z} \cdot \zeta-\bar{\zeta} \cdot z) / 4} I_{\infty}\left(\frac{b|z-\zeta|^{2}}{4}\right) .
\end{aligned}
$$

From these expressions, the lemma follows.

${ }^{1}$ Aftalion, A. and Helffer, B., "On mathematical models for Bose-Einstein condensates in optical lattices," Rev. Math. Phys. 21(2), 229-278 (2009).

${ }^{2}$ Agranovich, M. S., Sobolev Spaces and Their Generalizations Elliptic Problems in Domains with Smooth and Lipschitz Boundary (Moscow Center for Continuous Mathematical Education, 2013) (Russian).

${ }^{3}$ Agranovich, M. S., Katsenelenbaum, B. Z., Sivov, A. N., and Voitovich, N. N., Generalized Method of Eigenoscillations in Diffraction Theory (Wiley-VCH Verlag Berlin GmbH, Berlin, 1999) (Translated from the Russian manuscript by Vladimir Nazaikinskii).

${ }^{4}$ Birman, M. S.., “On the spectrum of singular boundary-value problems," Mat. Sb. (N.S.) 55(97), 125-174 (1961).

${ }^{5}$ Birman, M. S., "Perturbation of the spectrum of a singular elliptic operator under variation of the boundary and boundary conditions," Sov. Math. Dokl. 2, 326-328 (1961). 
${ }^{6}$ Birman, M. S. and Solomjak, M. Z., Spectral Theory of Selfadjoint Operators in Hilbert Space, Mathematics and Its Applications (Soviet Series) (D. Reidel Publishing Co., Dordrecht, 1987) (Translated from the 1980 Russian original by S. Khrushchëv and V. Peller).

${ }^{7}$ Erdélyi, A., Magnus, W., Oberhettinger, F., and Tricomi, F. G., Higher Transcendental Functions (McGraw-Hill Book Company, Inc., New York-Toronto-London, 1953), Vols. I and II. Based, in part, on notes left by Harry Bateman.

${ }^{8}$ Erdélyi, A., Magnus, W., Oberhettinger, F., and Tricomi, F. G., Tables of Integral Transforms (McGraw-Hill Book Company, Inc, New York-Toronto-London, 1954), Vol. I. Based, in part, on notes left by Harry Bateman.

${ }^{9}$ Filonov, N. and Pushnitski, A., "Spectral asymptotics of Pauli operators and orthogonal polynomials in complex domains," Commun. Math. Phys. 264(3), 759-772 (2006).

${ }^{10}$ Fournais, S. and Helffer, B., Spectral Methods in Surface Superconductivity, Progress in Nonlinear Differential Equations and Their Applications Vol. 77 (Birkhäuser, 2010).

${ }^{11}$ Fournais, S. and Kachmar, A., "On the energy of bound states for magnetic Schrödinger operators," J. London Math. Soc. 80(1), 233-255 (2009).

12 Frank, R. L., "On the asymptotic number of edge states for magnetic Schrödinger operators," Proc. London Math. Soc. 95(1), 1-19 (2007).

${ }^{13}$ Grisvard, P., Elliptic Problems in Nonsmooth Domains, Monographs and Studies in Mathematics Vol. 24 (Pitman Advanced Publishing Program, Boston, MA, 1985).

${ }^{14}$ Helffer, B. and Morame, A., "Magnetic bottles in connection with superconductivity," J. Funct. Anal. 185(2), 604-680 (2001).

${ }^{15}$ Hörmander, L., "The analysis of linear partial differential operators. III," in Classics in Mathematics, Pseudo-Differential Operators (Springer, Berlin, 2007), reprint of the 1994 edition.

${ }^{16}$ Hornberger, K. and Smilansky, U., "Magnetic edge states," Phys. Rep. 367(4), 249-385 (2002).

${ }^{17}$ Kachmar, A., "On the ground state energy for a magnetic Schrödinger operator and the effect of the DeGennes boundary condition," J. Math. Phys. 47(7), 072106 (2006), 32.

${ }^{18}$ Kachmar, A., "Weyl asymptotics for magnetic Schrödinger operators and de Gennes' boundary condition,” Rev. Math. Phys. 20(8), 901-932 (2008).

${ }^{19}$ Lions, J.-L. and Magenes, E., Non-Homogeneous Boundary Value Problems and Applications (Springer-Verlag, New York, 1972), Vol. I (Translated from the French by P. Kenneth, Die Grundlehren der mathematischen Wissenschaften, Band 181)

${ }^{20}$ Melgaard, M. and Rozenblum, G., "Eigenvalue asymptotics for weakly perturbed Dirac and Schrödinger operators with constant magnetic fields of full rank," Commun. Partial Differ. Equations 28(3-4), 697-736 (2003).

${ }^{21}$ Persson, M., "Eigenvalue asymptotics of the even-dimensional exterior Landau-Neumann Hamiltonian," Adv. Math. Phys. 2009, 873704

22 Pushnitski, A. and Rozenblum, G., "Eigenvalue clusters of the Landau Hamiltonian in the exterior of a compact domain," Doc. Math. 12, 569-586 (2007).

${ }^{23}$ Raikov, G. D. and Warzel, S., "Quasi-classical versus non-classical spectral asymptotics for magnetic Schrödinger operators with decreasing electric potentials," Rev. Math. Phys. 14(10), 1051-1072 (2002).

${ }^{24}$ Shubin, M. A., Pseudodifferential Operators and Spectral Theory, 2nd ed. (Springer-Verlag, Berlin, 2001) (Translated from the 1978 Russian original by Stig I. Andersson).

${ }^{25}$ Simon, B., Functional Integration and Quantum Physics, Pure and Applied Mathematics Vol. 86 (Academic Press, Inc. [Harcourt Brace Jovanovich Publishers], New York, 1979).

${ }^{26}$ Simon, B., Trace Ideals and Their Applications, 2nd ed. Mathematical Surveys and Monographs Vol. 120 (American Mathematical Society, Providence, RI, 2005).

${ }^{27}$ Taylor, M. E., Partial Differential Equations. II, Applied Mathematical Sciences Vol. 116 (Springer-Verlag, New York, 1996), Qualitative studies of linear equations. 